NISTIR 7883

\title{
Standardized Performance Testing Metrics for Optical Coordinate Measurement Systems
}

\author{
S.D. Phillips
}

http://dx.doi.org/10.6028/NIST.IR.7883

National Institute of Standards and Technology U.S. Department of Commerce 
NISTIR 7883

\title{
Standardized Performance Testing Metrics for Optical Coordinate Measurement Systems
}

\author{
S.D. Phillips
}

Semiconductor and Dimensional Division

Physical Measurement Laboratory

http://dx.doi.org/10.6028/NIST.IR.7883

May 2013

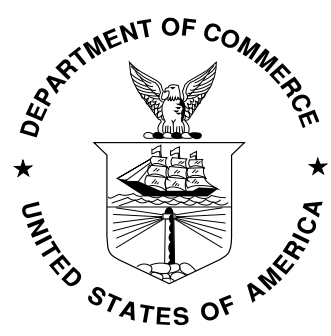

U.S. Department of Commerce Rebecca Blank, Acting Secretary

National Institute of Standards and Technology Patrick D. Gallagher, Under Secretary of Commerce for Standards and Technology and Director 


\title{
Standardized Performance Testing Metrics for Optical Coordinate Measurement Systems
}

\author{
S.D. Phillips \\ National Institute of Standards and Technology \\ Gaithersburg MD 20899
}

\begin{abstract}
Optical probing technology on coordinate measuring machines (CMMs) operates significantly differently from discrete point probing associated with tactile probing CMMs. Hence, manufacturers of this technology seek to use different testing artifacts for standardized performance testing. However, the standardized performance metrics which describe the CMM's measurement accuracy need to be comparable to other CMMs regardless of the probing technology. This paper examines several different metrics that can be computed from the test results of CMMs with optical probes, and these metrics are compared for equivalency to the canonical results specified by the ISO 10360 series of standards used for tactile CMMs. A derivation is presented which motivates a more detailed computer simulation study of nine different metrics. Of these nine, two metrics involve easily observable quantities for optical probing systems and are considered equivalent to the canonical performance test value.
\end{abstract}

Background: Coordinate measuring machines with tactile (i.e., contact) probing systems have a welldefined performance testing procedure codified in standards such as ISO 10360-2 [1] or its US counterpart ASME B89.4.10360.2 [2]. The three dimensional (3D) spatial measuring performance is specified by the $\operatorname{MPE}\left(E_{0}\right)^{1}$ value which is the maximum permissible error (MPE) of the " $E_{0}$ " parameter"; this describes the length measuring accuracy of the $\mathrm{CMM}$. In these standards, the performance test procedures (and hence the meaning of the $E$ value) are described in detail. The test includes 35 different measurements of calibrated length standards, each repeated three times, for a total of 105 observed length errors. There are several different formats in which the MPE specification can be stated. In this paper the MPE(E) specification is considered an upper bound for all possible length measurement errors (regardless of sign) for a CMM that is within its Original Equipment Manufacturer $(O E M)$ specified performance, i.e., $\operatorname{Max}|E| \leq \operatorname{MPE}(E)^{3}$. Each length measurement is specified as the point-to-point length derived from a single point coordinate recorded at each end of the calibrated standard, where the end surfaces are planar and the normal vectors to the two end surfaces are diametrically opposite, e.g., a gage block. This is taken as the canonical form of the measurement and the associated $\operatorname{Max}|E|$ value (of the 105 measurements) as its canonical test result value.

Over the last several years a plethora of new 3D CMMs have become available using various forms of optical probing technology; these include laser scanning systems, optical fringe projection systems, and $\mathrm{X}$-ray computer tomography systems. In many applications these systems can perform the same

\footnotetext{
${ }^{1}$ In the most recent ISO standards the notation is $E_{0, \text { MPE }}$ however for readability in this paper it is denoted $\operatorname{MPE}\left(E_{0}\right)$.

${ }^{2}$ The subscript associated with the $E$ value specifies the ram axis offset for the probing system; this offset depends on how the probe is mounted and is not of immediate concern for this paper so the subscript is not presented in the rest of this paper.

${ }^{3}$ In this paper the MPE is considered a constant, but a length dependent MPE is also a valid means of specifying the maximum error, i.e., there could be a different MPE value for each length of the artifact under testing.
} 
measurements as tactile CMMs, and hence users are faced with complex decisions regarding the capital equipment purchase of this equipment and the measurement application of this technology. One goal of standardized performance specifications ${ }^{4}$ is to make this decision more rigorous by allowing meaningful accuracy comparisons between different competing products. Consequently, developing a measurement performance specification that is comparable across multiple technologies is highly desirable.

Since optical probing systems are obviously noncontact, new standardized testing procedures are needed to specify these systems. However, the testing results (and hence the associated MPE specification) need to be closely comparable to that of $\operatorname{MPE}(E)$ for tactile CMMs in the sense that similar $\operatorname{MPE}(E)$ specifications across different technologically based CMMs will yield similar dimensional measurement accuracy for the same standardized measurand.

Tactile CMMs are typically tested in the canonical manner using calibrated artifacts such as gage blocks or step gages that have parallel planar opposing faces. While a direct comparison of tactile and optical measurement systems would involve measuring the same artifact in the same manner, optical CMM systems frequently have line of sight requirements that make traditional artifacts problematic. Additionally, tactile CMMs are designed to record discrete point coordinates and then move on to the next point location, while optical systems frequently record hundreds (or thousands) of points very rapidly within a single scan. Hence optical CMMs generally prefer extended targets such as the spheres on ball bars or ball plates because the spherical surface is observable from most orientations and the surface allows large numbers of points to be recorded. The terms bi-directional measurement and unidirectional measurement are used to refer to the detection of "size" errors. A ball bar measured in a center-to-center manner is a unidirectional measurement whereas if the ball bar measurement is the center-to-center length plus the sum of each sphere radius it is a bidirectional measurement because the measurement of sphere diameter is sensitive to detecting errors in the CMM's "effective probe size" ${ }^{\prime \prime}$; canonical testing artifacts such as gage blocks are measured in a bidirectional manner.

For reasons of economy and convenience, standardization committees seek testing methodology alternatives to the canonical procedure; in particular, to allow the measurement of many points on spherical targets yet yield a test value that is comparable to the canonical $E$ value result. Hence a form of "equivalent" testing metric is needed for the performance test results and the associated CMM accuracy specifications.

In the CMM performance testing sense, equivalence means that the test result - which in the canonical procedure is the maximum observed error, regardless of sign, of 105 bi-directionally measured lengths, is approximately the same ${ }^{6}$. Since the $E$ value test result is primarily designed to assess CMM volumetric measurement capability, e.g., to detect CMM structurally induced errors, Standards Committees

\footnotetext{
${ }^{4}$ Standardized specifications also lower costs for manufacturers by eliminating idiosyncratic user specified tests, and by aggregating economy of scale on testing artifacts and technician training.

${ }^{5}$ The term "effective" is used because even in the tactile probing case the proper stylus size may be different from its physical size to account for effects such as stylus bending; analogous effects also occur for optical probes.

${ }^{6}$ Test values from repeated " $E$ " tests on the same CMM rarely yield the exactly same value.
} 
designate the location and orientation of the 35 calibrated lengths for each type of $\mathrm{CMM}^{7}$ so that they appropriately capture these errors for the particular system under test.

\section{Performance Test Equivalency and Error Detection}

To employ the use of spherical artifacts in the $E$ test, the effect on various error sources - as detected in individual point coordinates - is considered first from a loose but intuitive model; later the quantitative simulation results will provide firm numerical results.

Consider a bi-directional measurement and a uni-directional measurement, as shown in Figure 1. The error sources associated with the length measurement can be classified into three categories:

Long range systematic errors: Geometrical errors in the CMM structure, thermal errors, etc.

Short range errors: repeatability problems, probing system errors such as anisotropic stylus bending and stylus out-of-roundness, short range scale errors, etc.

"Size" errors: an incorrect "effective probe size" as used by the CMM in length measurements.

Consider two measurements, one on a gage block in the canonical manner and one on a ball bar. Let the location of the two ends of the gage block be the same as the ball bar, i.e., the 3D coordinates of the sphere centers of the ball bar be the same as the coordinates of the gaging points of the gage block. Let the balls be small in the sense that they detect short range errors but are not sensitive to long range errors. Then the ball bar center-to-center distance will record the same long range errors as that of the canonical measurement. Hence the histogram of the long range errors -- shown schematically in the center of Figure 1 - will be the same for both cases.

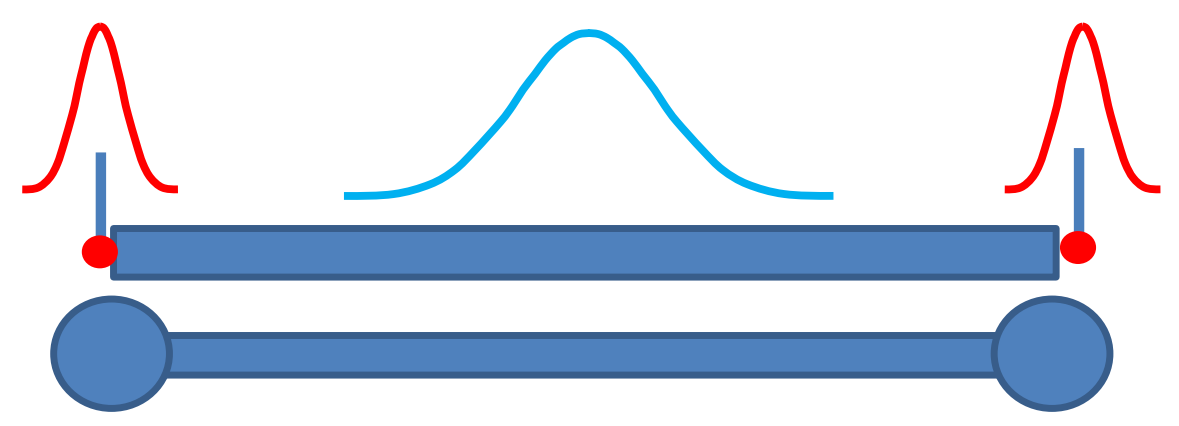

Figure 1: Gage block bi-directional measurement and ball bar uni-directional measurement

Let the balls be measured with a sufficient point density during the scanning process that the short range errors are significantly muted, i.e., "averaged out," hence these errors must be added back to obtain an equivalency with the canonical single point bi-directional measurement case. The short range errors for the canonical measurement are schematically shown above each stylus tip in Figure 1.

\footnotetext{
${ }^{7}$ For example, AACMM test artifact positions might be different from that of a Cartesian CMM but the intent with regard to error assessment is the same.
} 
It is also obvious that an error in the probing system's effective probe size directly adds in a one-to-one manner $^{8}$ to the sum of short range and long range errors for each length measurement in the canonical procedure. However, the uni-directional sphere center to sphere center case is insensitive to detecting this error. Hence it is clear that the error in the effective probe size ${ }^{9}$ must be added to the individual uni-directional length measurement. ${ }^{10}$

For each gage block measurement these three errors combine together to yield the observed error; this occurs for all 105 length measurements. It is useful to consider the 105 test measurements repeated a large number of times, each time yielding a slightly different histogram because both the long range and the short range errors are not perfectly repeatable and may also change with time. Similarly the size errors may change, e.g., due to probe requalification. The combination of all these histograms yields a distribution of values. Then the expected $E$ test result would be the $105^{\text {th }}$ order statistic from a sample of 105 absolute values from this distribution.

For two different performance tests to be metrologically equivalent, they must detect the same error sources and have similar sensitivities to these error sources; e.g., if the short range errors are increased such that one performance test result value increases by $\mathrm{X}$ micrometers, then the value obtained from the other performance test should similarly increase.

The long range errors are the same for both the gage block and the ball bar cases; hence this distribution is obtained directly for the observed ball bar center-to-center length errors.

The CMM's effective probe size error can be observed directly in the ball bar case if the balls are calibrated for diameter or alternatively from the size error observed in a calibrated test sphere measurement, e.g., the PS value obtained from the probe size test in [3, 4]. An upper bound on the PS value can be estimated from the OEM specifications: MPE(PS). This error arithmetically adds to each individual long range error. However, since the sign of the largest long range error and that of the size error may be different, it does not necessarily follow that the same test result value is obtained from the arithmetic addition of the size error and the largest (regardless of sign) center-to-center length error; see Annex A for additional details.

Since the short range errors have been mostly averaged out over the ball surfaces, they need to be included as follows. Consider selecting any position of the 105 canonical measurements (pick an element from the center-to-center error distribution), since the short range errors can be considered random, an element from their distribution at the right end and similarly at the left end. From a statistical viewpoint, since these are independent distributions, the variances will sum arithmetically; an estimate of the short range errors can be obtained from the ISO 10360-5 (or equivalent) sphere form test result, i.e., the $P F$ value [3]. For CMMs with tactile probing systems the $P F$ value is the range of residuals of a least-squares sphere fit of 25 discrete probing points located approximately uniformly

\footnotetext{
${ }^{8}$ For example, each micrometer of effective probe size error results in one micrometer error in the reported gage length.

${ }^{9}$ In Figure 1 this is the effective stylus tip size.

${ }^{10}$ Mathematically, there is a difference between adding the size error to an individual length measurement and adding it to the test result due to the use of order statistics in the determination of the test result; see Annex A.
} 
distributed on a hemisphere of a test sphere. For CMMs with optical probing systems a similar test has been proposed [4] where the 25 residuals of the sphere fit derived from 25 "representative points," each obtained from a small (non-overlapping) area on the test sphere surface.

\section{Equivalent Test Result Calculations}

First consider the case where the mean of the long range, short range, and probe size errors are all zero and the distributions are all Gaussian. The distribution of the 105 length errors can be considered the convolution of the input error distributions; hence the length errors are Gaussian distributed with mean zero with variance $\sigma_{\text {Errors }}$, this is just the sum of the variances of the input error distributions. Let $\beta$ be the number of standard deviations of a Gaussian distribution to yield the $105^{\text {th }}$ order statistic from a sample of 105 absolute value length errors; for Gaussian distributions $\beta$ is about 2.8. Noting that the long range errors are captured in the center-to-center length measurement allows the replacement of

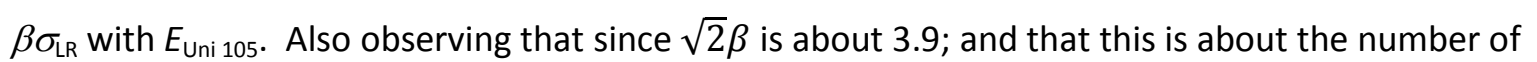
standard deviations of short range errors which is expected ${ }^{11}$ to produce the reported form error using the ISO 25 point test, i.e., the PF value; combining these observations results in Equation (1). The usefulness of Equation (1) is that $E_{\text {Uni } 105}$ (the largest observed error regardless of sign in the center-tocenter length of a calibrated ball bar) and the PF value (the form test result from [3] or [4]) are readily available test result values which can be combined to yield the equivalent bi-directional test result.

$$
E_{\mathrm{Bi} 105}=\beta \sigma_{\mathrm{Errors}}=\sqrt{\beta^{2} \sigma_{\mathrm{LR}}^{2}+2 \beta^{2} \sigma_{\mathrm{SR}}^{2}}=\sqrt{E_{\mathrm{Uni} 105}^{2}+2 \beta^{2} \sigma_{\mathrm{SR}}^{2}} \approx \sqrt{E_{\mathrm{Uni} 105}^{2}+P F^{2}}
$$

In general, the distribution for the canonical length measurement errors will be biased due to a combination of a bias in the long range errors, e.g., a global scale error in the CMM, or a bias arising from an effective probe size error. While the center-to-center length of a ball bar is sensitive to the bias in the long range errors, it is insensitive to the effective probe size error. Since $E_{\text {Uni } 105}$ is the largest observed error in the center-to-center distance, regardless of sign, this observed quantity will include the long range bias effect. To address the effective probe size error we note that the order statistic $\mathrm{E}_{105}$ associated with taking the maximum from 105 absolute values arising from a Gaussian distribution of mean $\mu$ and standard deviation $\sigma, \mathrm{N}[\mu, \sigma]$, is bounded as shown in Equation (2), where $\beta$ is the expected value of the $105^{\text {th }}$ order statistic of a sample of 105 absolute values taken from a $N[0,1]$ population; this is also the number of standard deviations from a $\mathrm{N}[0, \sigma]$ population needed to reach this order statistic.

$$
E_{105} \leq \beta \sigma+\mu
$$

A good estimate of the effective probe size error is the PS value obtained from an ISO probe test such as [3] or [4]. Using this Equation (1) becomes:

$$
E_{\mathrm{Bi} 105}<\approx \sqrt{E_{\mathrm{Uni} 105}^{2}+P F^{2}}+P S \quad \text { where }<\approx \text { denotes an approximate upper bound. }
$$

\footnotetext{
${ }^{11}$ For a Gaussian distribution of short range errors with standard deviation $\sigma$, and using the default ISO 10360-5 test sampling strategy, the expected form error is $3.7 \sigma$ and has a standard deviation of $0.7 \sigma$.
} 
In some testing situations, the ball bar will have spheres calibrated for their diameter and this will detect the effective probe size error as an error in the ball diameters. Hence the length composed of the center-to-center length plus the radius of ball 1 and the radius of ball 2 (as computed from a least squares sphere fit) denoted: $L_{U n i}+R_{1}+R_{2}$ is a useful quantity. Let $E_{U n i R R ~ 105}$ denote the largest of these 105 lengths regardless of sign; this quantity would be equivalent to the canonical measurement if the short range errors were zero. Assuming that the spheres are densely measured so that the short range errors average out, then accounting for the short range errors in a manner similar to Equation (1) yields the equivalent formulation in Equation (4):

$$
E_{\mathrm{Bi} 105}<\approx \sqrt{E_{\mathrm{UniRR} 105}^{2}+P F^{2}}
$$

\section{Computer Simulations}

The above derivations provide motivation to explore various proposed metrics that can be created from ball bar measurements and compared to the canonical test result for equivalency. To explore the implications of proposed methods such as Equations (3) and (4) a large number of different scenarios are computed. Each scenario is defined by a long range distribution $N\left[\mu_{L R}, \sigma_{L R}\right]$, and a short range distribution $N\left[\mu_{\text {Size/2 }}, \sigma_{S R}\right]$ which models the short range errors are expected to have mean zero, and the size error is a source of bias denoted $\mu_{\text {size/2 }}$ which is one half the size bias (the canonical artifacts are probed on each end and their sum yields the size bias). For a given scenario, the simulation involves an ISO 10360-5 test to evaluate the PS and PF values (using the default 25 point sampling strategy and a least squares fitting algorithm) and then the evaluation of 35 lengths each repeated three times. Each of the 35 lengths will have a different error drawn from the $N\left[\mu_{\mathrm{LR}}, \sigma_{\mathrm{LR}}\right]$ distribution, and each probing point associated with the three repeated measurements has a different error drawn from the $\mathrm{N}\left[\mu_{\mathrm{Size} / 2}, \sigma_{\mathrm{SR}}\right]$ distribution. A canonical measurement involves measuring the length with one probing point on each end of the length. The ball bar measurement involves taking many points (for convenience the 25 point ISO pattern is selected) on each ball. The long range error associated with the ball bar measurement is the same as that with the corresponding canonical length measurement ${ }^{12}$ but each probing point on the ball surface has a different error drawn from $N\left[\mu_{\text {size } / 2}, \sigma_{S R}\right]$. The canonical test result is computed and also the various proposed equivalent test results. This entire process is repeated 1000 times for a given scenario in order to create a large sample to test results.

To explore the different proposed methods of combining short and long range errors, a total of 81 different scenarios were examined. In particular, three levels of each bias and standard deviation were examined so that scenarios would cover the cases for each of these parameters being less than, comparable, and greater than each other parameters; $3^{4}=81$ scenarios. In the actual simulation the levels of $0.001,0.003$, and 0.010 were selected for both the biases and standard deviations, and the nominal length of the artifacts was 1 meter. However, the particular numerical values of these levels is not critical, only their relative values are significant for testing the proposed methods.

\footnotetext{
${ }^{12}$ The same long range error is used because physically the ball bar is located in the same position and orientation within the CMM as the canonical artifact.
} 
Nine different computations of test results based on ball bar measurements are considered and compared to the canonical case; the metrics are described below.

Metric 1: $\sqrt{E_{\mathrm{Uni} 105}^{2}+P F^{2}}+P S$ This is the same as Equation (3).

Metric 2: $\sqrt{E_{\text {Uni } 105}^{2}+\operatorname{MPE}(P F)^{2}}+\operatorname{MPE}(P S) \quad$ This is Metric 1 with the MPE values in place of the test result values for $P S$ and $P F$. The MPE values are defined as the mean value plus two standard deviations as evaluated from the 1000 cycles.

Metric 3: $\sqrt{E_{\mathrm{Uni}+\mathrm{PS} 105}^{2}+P F^{2}}$ This metric accounts for the size error by adding the PS value to each of the 105 center-to-center lengths and then determining the largest error regardless of sign, then this is combined in quadrature with the $P F$ value.

Metric 4: $\sqrt{E_{\mathrm{Uni} R \mathrm{RR} 105}^{2}+P F^{2}}$ This is Equation (4) which involves a ball bar with spheres calibrated for diameter

Metric 5: $\sqrt{E_{U n i+P S}^{2} 105+\operatorname{MPE}(P F)^{2}}$ This is Metric 4 with the MPE value in place of the particular cycle $P F$ value.

Metric 6: $\sqrt{E_{\mathrm{Uni}+\mathrm{PS} 105}^{2}+\mathrm{MPE}(P F)^{2}}$ This is Metric 3 with the MPE value in place of the particular cycle $P F$ value.

Metric 7: $E_{\text {Uni } 105}+|P S|+P F$ this metric arithmetically combines the center-to-center statistic with the absolute value of the $P S$ test result and the $P F$ test result.

Metric 8: $\quad E_{\text {Uni } 105}+\operatorname{MPE}(P S)+\operatorname{MPE}(P F)$ this is Metric 7 with the MPE values in place of the $P S$ and $P F$ test results.

Metric 9: $\operatorname{Max}\left[E_{\text {Uni } 105}+P S+P F\left|, E_{\text {Uni } 105}+P S-P F\right|\right]$ This metric selects the larger case (regardless of sign) of adding or subtracting the $P F$ value to the $E_{\mathrm{Uni} 105}$ and $P S$ values.

\section{Simulation Results}

The numerical results for the nine metrics and 81 scenarios are included in tables at the end of this paper. Since the central issue is the equivalency of a proposed metric to that of the canonical case, examining the mean of the difference between each proposed metric and the canonical result, scaled by the canonical, is useful; in the tables at the end of this paper this is denoted Mean[(Metric_X $-\mathrm{Bi}) / \mathrm{Bi}$ ], where Metric $X$ is the test result for the $X^{\text {th }}$ metric, and $\mathrm{Bi}$ is the test result for the canonical case. This quantity presents the difference between the proposed test result of metric $X$ and the canonical test result as a fraction of the canonical test value. Note that this difference in the two metrics is computed for each cycle of the 1000 cycles and then the mean of this quantity is computed for each scenario. This 
is done for all 81 scenarios and presented as a scatter chart in Figure 2 for all 9 different metrics. From Figure 2 it is clear that Metrics 1, 3, and 4 have, on average, test results that are within $10 \%$ of the corresponding canonical value. For comparison, if the quantity (Bi_1-Bi_2)/Bi_2 is examined, where Bi_1 and Bi_2 are just repeats of the canonical test, the standard deviation of this quantity is between 0.03 and 0.2 depending on the particular scenario; hence Metrics 1, 3, and 4 can be considered "equivalent" in the sense that their expected differences from the canonical case are no greater than the standard deviation of two canonical results similarly differenced and scaled.

Metrics 5 and 6 use the $\mathrm{MPE}(P F)$ value and not surprisingly the metrics overestimate relative to the canonical value by as much as $40 \%$. Metric 2 use both $\operatorname{MPE}(P S)$ and $\operatorname{MPE}(P F)$ and overestimates by as much as $80 \%$. Metrics 7, 8, 9 are arithmetic sums of test results, and these metrics have been discussed in Standards Committees as possible useful test results; all of these metrics include scenarios that overestimate by as much as $40 \%$.

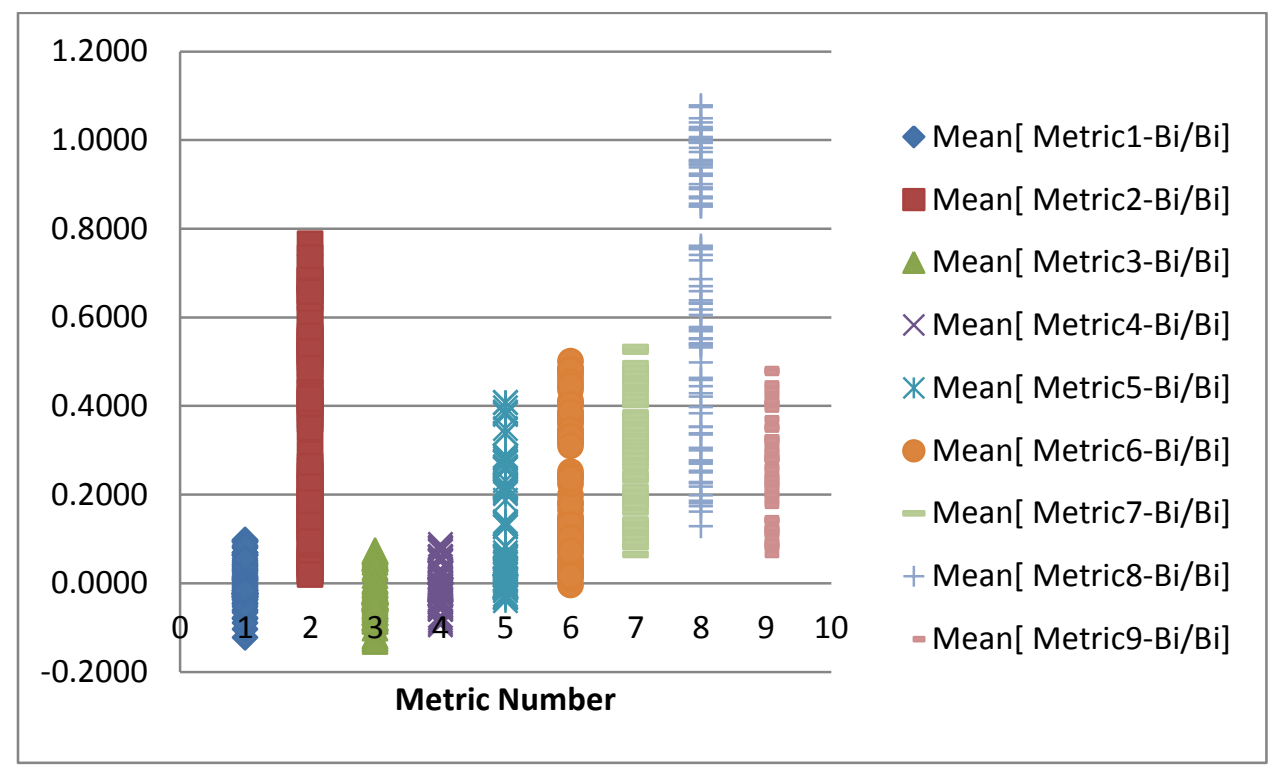

Figure 2: The mean difference of each of the nine computed metrics relative to the canonical result, as a fraction of the canonical result (the ratio is unitless).

A measure of the dispersion of the various metrics is shown in Figure 3, which displays the standard deviation of the 1000 test results per scenario divided by the corresponding canonical measurement standard deviation. Not surprisingly, those metrics that include MPEs, which are fixed quantities (for each scenario) show less variation. Metrics 3 and 4 show less variation than Metric 1 , perhaps because the size error is included in the length measurement before the order statistic is computed. 


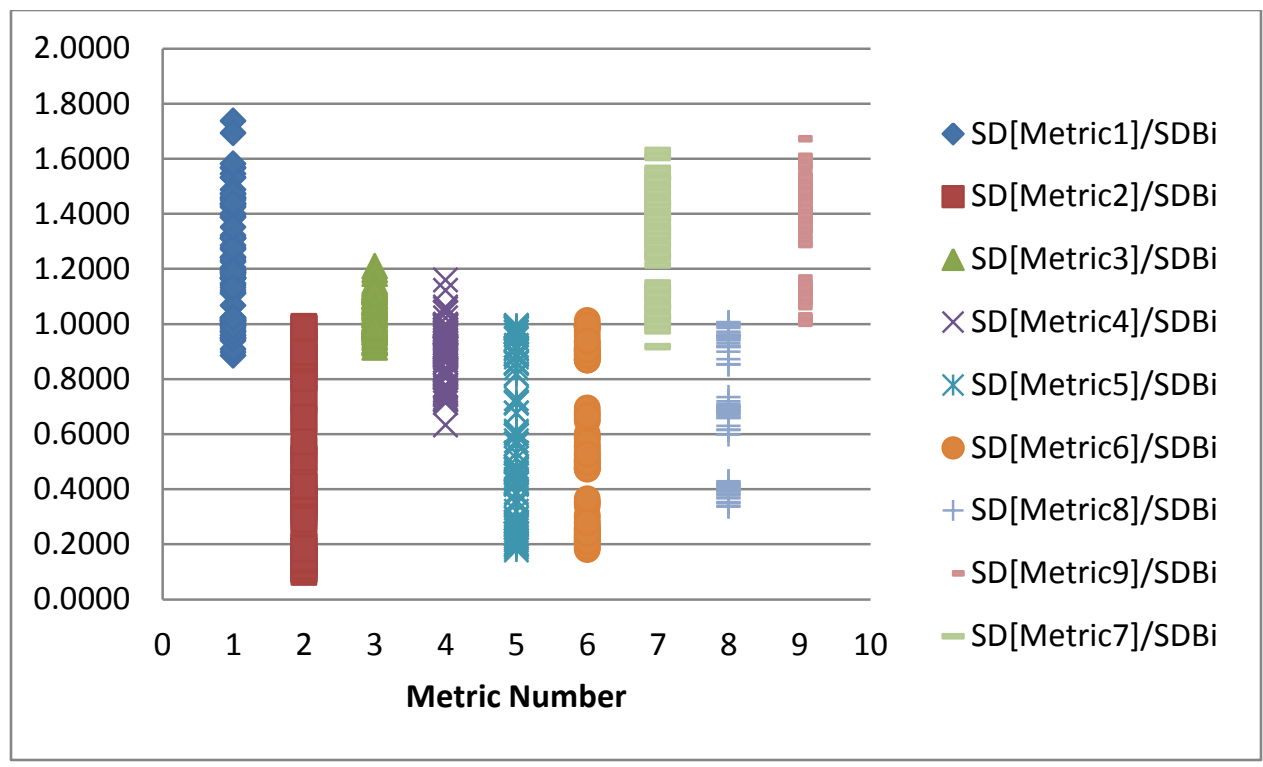

Figure 3: The ratio of the standard deviation of each of the nine computed metrics to that of the canonical case (the ratio is unitless).

\section{Summary}

Of the nine metrics examined in an effort to produce a test result based on ball bar measurements but equivalent to a (bi-directional) canonical test result (which is the $105^{\text {th }}$ order statistic of 105 length errors), only Metrics 3 and 4 appear reasonable for the case of Gaussianly distributed errors. If the probing errors are known to be non-Gaussian then this particular error distribution must be investigated before claims of equivalency can be asserted. 
Annex A: Discussion of order statistics

The test results as specified in CMM standards often employ order statistics, e.g., the largest observed error of 105 measurements regardless of sign. Some error sources may be fixed during all 105 measurements, such as the error associated with the effective probe size, so it is important to note that the addition of this fixed error to each individual length measurement results in a different value than adding this fixed error onto the order statistic found from the length measurements without the fixed error included.

The simplest way to see this is to consider a uniform distribution from -1 to +1 . Partition the distribution into 10 bins. The probability of drawing a value from one of the extreme bins (near \pm 1 ) is 2 out of 10 . Now draw a sample of size $\mathrm{N}$ elements and take their absolute values, with $\mathrm{N}$ just large enough that the Nth order statistic had the expectation in an extreme bin, e.g., 0.91. Consider adding a fixed constant, e.g., 0.5 , onto this result which yields 1.41 . Alternatively, add the 0.5 to all $\mathrm{N}$ values, this is equivalent to shifting the uniform distribution to between -0.5 and +1.5 , however now the probability of drawing a value in the extreme bin, i.e., 1.4 to 1.5 , is now just 1 out of 10 . For the case (above) where sample size of $\mathrm{N}$ was selected just large enough to yield an expected value in the extreme bin, the shifted distribution - with half the probability of drawing extreme values -- will cause the expectation value of the order statistic to yield a lower value. Letting the bin sizes become arbitrarily small, it is clear that the expectation value of the order statistic for the 0.5 to 1.5 distribution will be less than the -1 to 1 distribution regardless of the size of $\mathrm{N}$.

Another way to think of this is effect is that the absolute value requirement "folds" the negative portion of the distribution back into the positive side creating a new distribution. For the -1 to +1 case this becomes a uniform distribution from 0 to +1 , but for the -0.5 to +1.5 case it, the distribution becomes stepped, with the probability density being twice as large for the region 0 to 0.5 as in the region 0.5 to 1.5; not surprisingly changing the distribution changes the expectation value of the order statistics.

\section{References}

1 ISO 10360-2:2009(E) Geometrical product specifications (GPS) - Acceptance and reverification tests for coordinate measuring machines (CMM) - Part 2: CMMs used for measuring linear dimensions

2 ASME B89.4.10360.2-2008 Acceptance Test and Reverification Test for Coordinate Measuring Machines (CMMs) - Part 2: CMMs Used for Measuring Linear Dimensions, 2008; www.asme.org

3 ISO 10360-5: 2010 (E) Geometrical product specifications (GPS) - Acceptance and reverification tests for coordinate measuring machines (CMM) - Part 5: CMMs using single and multiple stylus contacting probing systems

4 ISO $2^{\text {nd }}$ DIS 10360-8: Geometrical Product Specifications (GPS) - Acceptance and reverification tests for coordinate measuring machines (CMM) - Part 8: CMMs with optical distance sensors (dated 2012-03-13). 
11

\begin{tabular}{|c|c|c|c|c|c|c|c|c|c|c|c|}
\hline Scenario number & 1 & 2 & 3 & 4 & 5 & 6 & 7 & 8 & 9 & 10 & 11 \\
\hline IongRangeErrorDist & $\mathrm{N}[0 ., 0.001]$ & $\mathrm{N}[0 ., 0.001]$ & $\mathrm{N}[0 ., 0.001]$ & $\mathrm{N}[0 ., 0.003]$ & $\mathrm{N}[0 ., 0.003]$ & $\mathrm{N}[0 ., 0.003]$ & $\mathrm{N}[0 ., 0.010]$ & $\mathrm{N}[0 ., 0.010]$ & $\mathrm{N}[0 ., 0.010]$ & $\mathrm{N}[0 ., 0.001]$ & $\mathrm{N}[0 ., 0.001]$ \\
\hline probeRndErrorDist & $\mathrm{N}[0 ., 0.001]$ & $\mathrm{N}[0 ., 0.003]$ & $\mathrm{N}[0 ., 0.010]$ & $\mathrm{N}[0 ., 0.001]$ & $\mathrm{N}[0 ., 0.003]$ & $\mathrm{N}[0 ., 0.010]$ & $\mathrm{N}[0 ., 0.001]$ & $\mathrm{N}[0 ., 0.003]$ & $\mathrm{N}[0 ., 0.010]$ & $\mathrm{N}[0.0015,0.001]$ & $\mathrm{N}[0.0015,0.003]$ \\
\hline Mean[Bi] & 0.0048 & 0.0120 & 0.0391 & 0.0087 & 0.0143 & 0.0400 & 0.0250 & 0.0287 & 0.0477 & 0.0073 & 0.0140 \\
\hline stdDevBi & 0.0007 & 0.0018 & 0.0055 & 0.0013 & 0.0020 & 0.0058 & 0.0043 & 0.0046 & 0.0069 & 0.0007 & 0.0018 \\
\hline Mean[Metric1] & 0.0047 & 0.0121 & 0.0395 & 0.0084 & 0.0141 & 0.0399 & 0.0244 & 0.0276 & 0.0474 & 0.0077 & 0.0151 \\
\hline SD[Metric1] & 0.0009 & 0.0027 & 0.0095 & 0.0014 & 0.0027 & 0.0089 & 0.0044 & 0.0045 & 0.0095 & 0.0009 & 0.0026 \\
\hline Mean[Metric1-Bi] & 0.0000 & 0.0001 & 0.0004 & -0.0003 & -0.0001 & 0.0000 & -0.0006 & $\mid-0.0011$ & -0.0003 & 0.0004 & 0.0011 \\
\hline SD[Metric1-Bi] & 0.0011 & 0.0032 & 0.0111 & 0.0012 & 0.0033 & 0.0111 & 0.0013 & 0.0034 & 0.0113 & 0.0011 & 0.0031 \\
\hline Mean[Metric1-Bi/Bi] & 0.0141 & 0.0269 & 0.0309 & -0.0249 & 0.0087 & 0.0217 & -0.0239 & -0.0314 & 0.0120 & 0.0642 & 0.0969 \\
\hline SD[ Metric1-Bi/Bi] & 0.2371 & 0.2674 & 0.2885 & 0.1347 & 0.2370 & 0.2761 & 0.0521 & 0.1187 & 0.2410 & 0.1585 & 0.2320 \\
\hline SD[Metric1]/SDBi & 1.3201 & 1.4592 & 1.7367 & 1.0129 & 1.3114 & 1.5317 & 1.0184 & 0.9853 & 1.3871 & 1.1874 & 1.4493 \\
\hline SD[Metric1-Bi]/SDBi & 1.6120 & 1.7369 & 2.0217 & 0.8622 & 1.6206 & 1.9062 & 0.2907 & 0.7450 & 1.6468 & 1.5294 & 1.7603 \\
\hline Mean[Metric2] & 0.0072 & 0.0199 & 0.0657 & 0.0105 & 0.0213 & 0.0657 & 0.0260 & 0.0336 & 0.0721 & 0.0101 & 0.0227 \\
\hline SD[Metric2] & 0.0003 & 0.0002 & 0.0006 & 0.0011 & 0.0008 & 0.0008 & 0.0043 & 0.0038 & 0.0025 & 0.0002 & 0.0002 \\
\hline Mean[Metric2-Bi] & 0.0024 & 0.0078 & 0.0266 & 0.0018 & 0.0070 & 0.0257 & 0.0010 & 0.0048 & 0.0244 & 0.0028 & 0.0086 \\
\hline SD[Metric2-Bi] & 0.0007 & 0.0018 & 0.0054 & 0.0009 & 0.0021 & 0.0059 & 0.0010 & 0.0028 & 0.0067 & 0.0008 & 0.0018 \\
\hline Mean[Metric2-Bi/Bi] & 0.5374 & 0.6852 & 0.7118 & 0.2203 & 0.5172 & 0.6773 & 0.0439 & 0.1814 & 0.5411 & 0.3926 & 0.6423 \\
\hline SD[ Metric2-Bi/Bi] & 0.2128 & 0.2461 & 0.2311 & 0.1289 & 0.2131 & 0.2344 & 0.0454 & 0.1159 & 0.2107 & 0.1378 & 0.2039 \\
\hline SD[Metric2]/SDBi & 0.3656 & 0.1292 & 0.1043 & 0.8043 & 0.3687 & 0.1313 & 0.9823 & 0.8356 & 0.3687 & 0.3248 & 0.1376 \\
\hline SD[Metric2-Bi]/SDBi & 0.9987 & 1.0063 & 0.9920 & 0.6819 & 1.0218 & 1.0102 & 0.2409 & 0.6036 & 0.9727 & 1.0137 & 1.0135 \\
\hline Mean[Metric3] & 0.0049 & 0.0127 & 0.0411 & 0.0086 & 0.0148 & 0.0418 & 0.0245 & 0.0281 & 0.0494 & 0.0068 & 0.0136 \\
\hline SD[Metric3] & 0.0006 & 0.0020 & 0.0066 & 0.0013 & 0.0019 & 0.0066 & 0.0043 & 0.0043 & 0.0068 & 0.0008 & 0.0019 \\
\hline Mean[Metric3-Bi] & 0.0002 & 0.0006 & 0.0020 & -0.0001 & 0.0005 & 0.0019 & -0.0005 & -0.0006 & 0.0018 & -0.0005 & -0.0005 \\
\hline SD[Metric3-Bi] & 0.0009 & 0.0027 & 0.0084 & 0.0011 & 0.0028 & 0.0091 & 0.0012 & 0.0032 & 0.0090 & 0.0010 & 0.0026 \\
\hline Mean[ Metric3-Bi/Bi] & 0.0580 & 0.0730 & 0.0704 & -0.0054 & 0.0556 & 0.0690 & -0.0199 & -0.0130 & 0.0558 & -0.0669 & -0.0177 \\
\hline SD[ Metric3-Bi/Bi] & 0.1963 & 0.2277 & 0.2216 & 0.1247 & 0.2040 & 0.2322 & 0.0479 & 0.1134 & 0.1993 & 0.1325 & 0.1860 \\
\hline SD[Metric3]/SDBi & 0.9296 & 1.0820 & 1.2094 & 0.9372 & 0.9489 & 1.1348 & 0.9922 & 0.9322 & 0.9906 & 1.0449 & 1.0977 \\
\hline SD[Metric3-Bi]/SDBi & 1.3234 & 1.4574 & 1.5313 & 0.8076 & 1.3789 & 1.5682 & 0.2712 & 0.7058 & 1.3175 & 1.3576 & 1.4766 \\
\hline Mean[Metric4] & 0.0049 & 0.0127 & 0.0414 & 0.0087 & 0.0147 & 0.0420 & 0.0246 & 0.0284 & 0.0491 & 0.0070 & 0.0141 \\
\hline SD[Metric4] & 0.0006 & 0.0019 & 0.0063 & 0.0012 & 0.0018 & 0.0061 & 0.0043 & 0.0042 & 0.0063 & 0.0006 & 0.0017 \\
\hline Mean[Metric4-Bi] & 0.0002 & 0.0007 & 0.0023 & -0.0001 & 0.0004 & 0.0020 & -0.0004 & -0.0004 & 0.0015 & -0.0003 & 0.0001 \\
\hline SD[Metric4-Bi] & 0.0009 & 0.0026 & 0.0082 & 0.0010 & 0.0027 & 0.0088 & 0.0011 & 0.0031 & 0.0087 & 0.0009 & 0.0024 \\
\hline Mean[ Metric4-Bi/Bi] & 0.0517 & 0.0784 & 0.0780 & 0.0025 & 0.0498 & 0.0735 & -0.0159 & -0.0050 & 0.0493 & -0.0365 & 0.0223 \\
\hline SD[ Metric4-Bi/Bi] & 0.1901 & 0.2219 & 0.2184 & 0.1166 & 0.1909 & 0.2242 & 0.0448 & 0.1074 & 0.1909 & 0.1134 & 0.1751 \\
\hline SD[Metric4]/SDBi & 0.8845 & 1.0215 & 1.1592 & 0.9053 & 0.8675 & 1.0551 & 0.9923 & 0.9084 & 0.9206 & 0.7738 & 0.9681 \\
\hline SD[Metric4-Bi]/SDBi & 1.2846 & 1.4118 & 1.5013 & 0.7446 & 1.2964 & 1.5070 & 0.2502 & 0.6657 & 1.2756 & 1.1656 & 1.3732 \\
\hline Mean[Metric5] & 0.0060 & 0.0164 & 0.0540 & 0.0093 & 0.0177 & 0.0543 & 0.0248 & 0.0302 & 0.0601 & 0.0078 & 0.0174 \\
\hline SD[Metric5] & 0.0003 & 0.0004 & 0.0010 & 0.0011 & 0.0009 & 0.0011 & 0.0043 & 0.0039 & 0.0029 & 0.0004 & 0.0005 \\
\hline Mean[Metric5-Bi] & 0.0012 & 0.0044 & 0.0149 & 0.0006 & 0.0034 & 0.0143 & -0.0002 & 0.0015 & 0.0124 & 0.0004 & 0.0034 \\
\hline SD[Metric5-Bi] & 0.0007 & 0.0019 & 0.0055 & 0.0009 & 0.0021 & 0.0059 & 0.0011 & 0.0029 & 0.0068 & 0.0008 & 0.0018 \\
\hline Mean[ Metric5-Bi/Bi] & 0.2762 & 0.3951 & 0.4078 & 0.0827 & 0.2629 & 0.3862 & -0.0060 & 0.0617 & 0.2839 & 0.0679 & 0.2594 \\
\hline SD[ Metric5-Bi/Bi] & 0.1794 & 0.2055 & 0.1914 & 0.1157 & 0.1786 & 0.1945 & 0.0445 & 0.1083 & 0.1776 & 0.1082 & 0.1598 \\
\hline SD[Metric5]/SDBi & 0.4191 & 0.2047 & 0.1881 & 0.8183 & 0.4160 & 0.1959 & 0.9829 & 0.8391 & 0.4262 & 0.5608 & 0.2926 \\
\hline SD[Metric5-Bi]/SDBi & 1.0138 & 1.0167 & 1.0020 & 0.6950 & 1.0272 & 1.0112 & 0.2483 & 0.6386 & 0.9959 & 1.0371 & 1.0400 \\
\hline Mean[Metric6] & 0.0064 & 0.0174 & 0.0569 & 0.0097 & 0.0191 & 0.0574 & 0.0248 & 0.0310 & 0.0647 & 0.0086 & 0.0189 \\
\hline SD[Metric6] & 0.0003 & 0.0004 & 0.0010 & 0.0012 & 0.0010 & 0.0012 & 0.0043 & 0.0041 & 0.0033 & 0.0004 & 0.0005 \\
\hline Mean[Metric6-Bi] & 0.0017 & 0.0053 & 0.0178 & 0.0010 & 0.0048 & 0.0174 & -0.0002 & 0.0023 & 0.0170 & 0.0012 & 0.0048 \\
\hline SD[Metric6-Bi] & 0.0007 & 0.0019 & 0.0055 & 0.0012 & 0.0022 & 0.0059 & 0.0016 & 0.0037 & 0.0070 & 0.0008 & 0.0018 \\
\hline Mean[ Metric6-Bi/Bi] & 0.3743 & 0.4729 & 0.4826 & 0.1264 & 0.3594 & 0.4641 & -0.0040 & 0.0924 & 0.3830 & 0.1806 & 0.3670 \\
\hline SD[ Metric6-Bi/Bi] & 0.1946 & 0.2178 & 0.2004 & 0.1460 & 0.1950 & 0.2061 & 0.0639 & 0.1347 & 0.1930 & 0.1165 & 0.1731 \\
\hline SD[Metric6]/SDBi & 0.4836 & 0.2227 & 0.1892 & 0.8750 & 0.4978 & 0.2111 & 0.9881 & 0.8924 & 0.4775 & 0.5537 & 0.2751 \\
\hline SD[Metric6-Bi]/SDBi & 1.0420 & 1.0282 & 1.0012 & 0.8735 & 1.0577 & 1.0221 & 0.3589 & 0.7959 & 1.0186 & 1.0232 & 1.0395 \\
\hline Mean[Metric7] & 0.0071 & 0.0175 & 0.0559 & 0.0118 & 0.0214 & 0.0576 & 0.0283 & 0.0379 & 0.0714 & 0.0096 & 0.0191 \\
\hline SD[Metric7] & 0.0009 & 0.0026 & 0.0083 & 0.0015 & 0.0027 & 0.0085 & 0.0044 & 0.0050 & 0.0095 & 0.0010 & 0.0027 \\
\hline Mean[Metric7-Bi] & 0.0024 & 0.0054 & 0.0167 & 0.0031 & 0.0071 & 0.0176 & 0.0033 & 0.0092 & 0.0237 & 0.0023 & 0.0051 \\
\hline SD[Metric7-Bi] & 0.0011 & 0.0031 & 0.0096 & 0.0013 & 0.0034 & 0.0106 & 0.0013 & 0.0036 & 0.0109 & 0.0013 & 0.0032 \\
\hline Mean[ Metric7-Bi/Bi] & 0.5299 & 0.4835 & 0.4529 & 0.3681 & 0.5278 & 0.4713 & 0.1381 & 0.3331 & 0.5232 & 0.3224 & 0.3841 \\
\hline SD[ Metric7-Bi/Bi] & 0.2813 & 0.3036 & 0.2837 & 0.1731 & 0.2877 & 0.3059 & 0.0628 & 0.1530 & 0.2810 & 0.1892 & 0.2620 \\
\hline SD[Metric7]/SDBi & 1.3063 & 1.3988 & 1.5228 & 1.1204 & 1.3163 & 1.4660 & 1.0178 & 1.0929 & 1.3862 & 1.3964 & 1.5269 \\
\hline SD[Metric7-Bi]/SDBi & 1.5985 & 1.7014 & 1.7568 & 0.9315 & 1.6447 & 1.8179 & 0.3071 & 0.7907 & 1.5921 & 1.6814 & 1.8272 \\
\hline Mean[Metric8] & 0.0093 & 0.0240 & 0.0779 & 0.0140 & 0.0276 & 0.0792 & 0.0305 & 0.0445 & 0.0935 & 0.0122 & 0.0268 \\
\hline SD[Metric8] & 0.0005 & 0.0007 & 0.0020 & 0.0013 & 0.0014 & 0.0024 & 0.0044 & 0.0045 & 0.0048 & 0.0005 & 0.0007 \\
\hline Mean[Metric8-Bi] & 0.0045 & 0.0120 & 0.0388 & 0.0053 & 0.0134 & 0.0393 & 0.0055 & 0.0158 & 0.0459 & 0.0049 & 0.0128 \\
\hline SD[Metric8-Bi] & 0.0008 & 0.0020 & 0.0056 & 0.0010 & 0.0023 & 0.0063 & 0.0011 & 0.0029 & 0.0073 & 0.0008 & 0.0019 \\
\hline Mean[ Metric8-Bi/Bi] & 0.9930 & 1.0390 & 1.0285 & 0.6304 & 0.9724 & 1.0223 & 0.2277 & 0.5681 & 0.9979 & 0.6860 & 0.9450 \\
\hline SD[ Metric8-Bi/Bi] & 0.2795 & 0.3021 & 0.2740 & 0.1747 & 0.2823 & 0.2880 & 0.0616 & 0.1584 & 0.2745 & 0.1708 & 0.2474 \\
\hline SD[Metric8]/SDBi & 0.6974 & 0.3943 & 0.3684 & 0.9592 & 0.6946 & 0.4110 & 1.0027 & 0.9706 & 0.6988 & 0.6154 & 0.4155 \\
\hline SD[Metric8-Bi]/SDBi & 1.1064 & 1.0693 & 1.0240 & 0.7161 & 1.1428 & 1.0842 & 0.2430 & 0.6251 & 1.0607 & 1.1086 & 1.0936 \\
\hline Mean[Metric9] & 0.0069 & 0.0171 & 0.0548 & 0.0114 & 0.0207 & 0.0564 & 0.0278 & 0.0368 & 0.0692 & 0.0093 & 0.0187 \\
\hline SD[Metric9] & 0.0009 & 0.0025 & 0.0083 & 0.0015 & 0.0027 & 0.0084 & 0.0044 & 0.0050 & 0.0094 & 0.0011 & 0.0027 \\
\hline Mean[Metric9-Bi] & 0.0022 & 0.0050 & 0.0157 & 0.0027 & 0.0064 & 0.0164 & 0.0028 & 0.0081 & 0.0216 & 0.0020 & 0.0047 \\
\hline SD[Metric9-Bi] & 0.0011 & 0.0031 & 0.0096 & 0.0013 & 0.0034 & 0.0105 & 0.0013 & 0.0038 & 0.0108 & 0.0012 & 0.0032 \\
\hline Mean[ Metric9-Bi/Bi] & 0.4818 & 0.4493 & 0.4255 & 0.3228 & 0.4794 & 0.4417 & 0.1184 & 0.2942 & 0.4783 & 0.2820 & 0.3551 \\
\hline SD[ Metric9-Bi/Bi] & 0.2724 & 0.2975 & 0.2821 & 0.1734 & 0.2834 & 0.3023 & 0.0613 & 0.1568 & 0.2757 & 0.1823 & 0.2574 \\
\hline SD[Metric9]/SDBi & 1.2868 & 1.3765 & 1.5129 & 1.1229 & 1.3188 & 1.4454 & 1.0161 & 1.0842 & 1.3648 & 1.4140 & 1.5102 \\
\hline SD[Metric9-Bi]/SDBi & 1.5757 & 1.6847 & 1.7592 & 0.9619 & 1.6494 & 1.8105 & 0.3104 & 0.8274 & 1.5824 & 1.6455 & 1.8080 \\
\hline formMean & 0.0036 & 0.0110 & 0.0365 & 0.0037 & 0.0109 & 0.0364 & 0.0037 & 0.0110 & 0.0363 & 0.0036 & 0.0110 \\
\hline formStdDev & 0.0007 & 0.0021 & 0.0070 & 0.0007 & 0.0019 & 0.0069 & 0.0007 & 0.0021 & 0.0070 & 0.0007 & 0.0020 \\
\hline Points per sphere & 25 & 25 & 25 & 25 & 25 & 25 & 25 & 25 & 25 & 25 & 25 \\
\hline Scenario number & 1 & 2 & 3 & 4 & 5 & 6 & 7 & 8 & 9 & 10 & 11 \\
\hline
\end{tabular}


12

\begin{tabular}{|c|c|c|c|c|c|c|c|c|c|c|}
\hline Scenario number & 12 & 13 & 14 & 15 & 16 & 17 & 18 & 19 & 20 & 21 \\
\hline IongRangeErrorDist & $\mathrm{N}[0 ., 0.001]$ & $\mathrm{N}[0 ., 0.003]$ & $\mathrm{N}[0 ., 0.003]$ & $\mathrm{N}[0 ., 0.003]$ & $\mathrm{N}[0 ., 0.010]$ & $\mathrm{N}[0 ., 0.010]$ & $\mathrm{N}[0 ., 0.010]$ & $\mathrm{N}[0 ., 0.001]$ & $\mathrm{N}[0,0.001]$ & $\mathrm{N}[0 ., 0.001]$ \\
\hline probeRndErrorDist & $\mathrm{N}[0.0015,0.010]$ & $\mathrm{N}[0.0015,0.001]$ & $\mathrm{N}[0.0015,0.003]$ & $\mathrm{N}[0.0015,0.010]$ & $\mathrm{N}[0.0015,0.001]$ & $\mathrm{N}[0.0015,0.003]$ & $\mathrm{N}[0.0015,0.010]$ & $\mathrm{N}[0.005,0.001]$ & $\mathrm{N}[0.005,0.003]$ & $\mathrm{N}[0.005,0.010]$ \\
\hline Mean[Bi] & 0.0398 & 0.0109 & 0.0161 & 0.0407 & 0.0261 & 0.0297 & 0.0483 & 0.0144 & 0.0210 & 0.0456 \\
\hline stdDevBi & 0.0056 & 0.0015 & 0.0022 & 0.0059 & 0.0046 & 0.0048 & 0.0070 & 0.0008 & 0.0019 & 0.0060 \\
\hline Mean[Metric1] & 0.0427 & 0.0115 & 0.0173 & 0.0431 & 0.0275 & 0.0308 & 0.0508 & 0.0147 & 0.0221 & 0.0492 \\
\hline SD[Metric1] & 0.0094 & 0.0014 & 0.0027 & 0.0090 & 0.0044 & 0.0047 & 0.0091 & 0.0009 & 0.0028 & 0.0094 \\
\hline Mean[Metric1-Bi] & 0.0029 & 0.0006 & 0.0012 & 0.0025 & 0.0014 & 0.0011 & 0.0024 & 0.0004 & 0.0011 & 0.0036 \\
\hline SD[Metric1-Bi] & 0.0108 & 0.0016 & 0.0034 & 0.0108 & 0.0028 & 0.0040 & 0.0109 & 0.0011 & 0.0034 & 0.0108 \\
\hline Mean[ Metric1-Bi/Bi] & 0.0930 & 0.0678 & 0.0937 & 0.0822 & 0.0637 & 0.0467 & 0.0688 & 0.0290 & 0.0618 & 0.0952 \\
\hline SD[ Metric1-Bi/Bi] & 0.2838 & 0.1640 & 0.2224 & 0.2734 & 0.1169 & 0.1417 & 0.2342 & 0.0787 & 0.1645 & 0.2450 \\
\hline SD[Metric1]/SDBi & 1.6936 & 0.9398 & 1.2424 & 1.5442 & 0.9591 & 0.9716 & 1.3105 & 1.2260 & 1.4589 & 1.5655 \\
\hline SD[Metric1-Bi]/SDBi & 1.9442 & 1.0742 & 1.5768 & 1.8444 & 0.6078 & 0.8312 & 1.5674 & 1.4879 & 1.7917 & 1.8071 \\
\hline Mean[Metric2] & 0.0690 & 0.0135 & 0.0244 & 0.0692 & 0.0291 & 0.0364 & 0.0757 & 0.0172 & 0.0298 & 0.0754 \\
\hline SD[Metric2] & 0.0006 & 0.0011 & 0.0008 & 0.0008 & 0.0043 & 0.0039 & 0.0026 & 0.0003 & 0.0002 & 0.0006 \\
\hline Mean[Metric2-Bi] & 0.0292 & 0.0026 & 0.0083 & 0.0285 & 0.0030 & 0.0067 & 0.0274 & 0.0029 & 0.0089 & 0.0298 \\
\hline SD[Metric2-Bi] & 0.0056 & 0.0014 & 0.0022 & 0.0059 & 0.0027 & 0.0034 & 0.0069 & 0.0007 & 0.0019 & 0.0060 \\
\hline Mean[ Metric2-Bi/Bi] & 0.7652 & 0.2531 & 0.5435 & 0.7345 & 0.1267 & 0.2427 & 0.5967 & 0.2027 & 0.4333 & 0.6814 \\
\hline SD[ Metric2-Bi/Bi] & 0.2403 & 0.1608 & 0.2043 & 0.2388 & 0.1180 & 0.1433 & 0.2215 & 0.0609 & 0.1264 & 0.2143 \\
\hline SD[Metric2]/SDBi & 0.1086 & 0.7312 & 0.3465 & 0.1343 & 0.9344 & 0.8139 & 0.3704 & 0.3482 & 0.1300 & 0.0967 \\
\hline SD[Metric2-Bi]/SDBi & 1.0056 & 0.9135 & 1.0035 & 1.0008 & 0.5798 & 0.7022 & 0.9945 & 0.9903 & 1.0108 & 1.0051 \\
\hline Mean[Metric3] & 0.0418 & 0.0105 & 0.0159 & 0.0424 & 0.0255 & 0.0290 & 0.0502 & 0.0132 & 0.0183 & 0.0437 \\
\hline SD[Metric3] & 0.0066 & 0.0015 & 0.0022 & 0.0064 & 0.0045 & 0.0047 & 0.0068 & 0.0009 & 0.0021 & 0.0069 \\
\hline Mean[Metric3-Bi] & 0.0020 & -0.0004 & -0.0002 & 0.0017 & -0.0006 & -0.0007 & 0.0019 & -0.0012 & -0.0027 & -0.0019 \\
\hline SD[Metric3-Bi] & 0.0086 & 0.0012 & 0.0029 & 0.0087 & 0.0013 & 0.0034 & 0.0090 & 0.0010 & 0.0029 & 0.0089 \\
\hline Mean[ Metric3-Bi/Bi] & 0.0703 & -0.0345 & 0.0031 & 0.0632 & -0.0216 & -0.0171 & 0.0575 & -0.0784 & -0.1198 & -0.0273 \\
\hline SD[ Metric3-Bi/Bi] & 0.2240 & 0.1067 & 0.1794 & 0.2183 & 0.0507 & 0.1153 & 0.1927 & 0.0668 & 0.1294 & 0.1937 \\
\hline SD[Metric3]/SDBi & 1.1817 & 0.9921 & 1.0019 & 1.0971 & 0.9827 & 0.9681 & 0.9737 & 1.1329 & 1.1223 & 1.1523 \\
\hline SD[Metric3-Bi]/SDBi & 1.5375 & 0.7733 & 1.3201 & 1.4900 & 0.2823 & 0.7122 & 1.2913 & 1.3209 & 1.5140 & 1.4939 \\
\hline Mean[Metric4] & 0.0425 & 0.0107 & 0.0163 & 0.0431 & 0.0256 & 0.0293 & 0.0502 & 0.0135 & 0.0193 & 0.0458 \\
\hline SD[Metric4] & 0.0062 & 0.0014 & 0.0018 & 0.0061 & 0.0045 & 0.0046 & 0.0064 & 0.0006 & 0.0014 & 0.0058 \\
\hline Mean[Metric4-Bi] & 0.0026 & -0.0003 & 0.0002 & 0.0024 & -0.0005 & -0.0005 & 0.0019 & -0.0009 & -0.0017 & 0.0002 \\
\hline SD[Metric4-Bi] & 0.0083 & 0.0010 & 0.0026 & 0.0084 & 0.0012 & 0.0030 & 0.0088 & 0.0008 & 0.0024 & 0.0083 \\
\hline Mean[ Metric4-Bi/Bi] & 0.0864 & -0.0186 & 0.0269 & 0.0800 & -0.0170 & -0.0091 & 0.0577 & -0.0598 & -0.0743 & 0.0214 \\
\hline SD[ Metric4-Bi/Bi] & 0.2197 & 0.0919 & 0.1615 & 0.2118 & 0.0458 & 0.1019 & 0.1904 & 0.0554 & 0.1070 & 0.1828 \\
\hline SD[Metric4]/SDBi & 1.1218 & 0.9134 & 0.8306 & 1.0393 & 0.9787 & 0.9512 & 0.9191 & 0.7773 & 0.7569 & 0.9669 \\
\hline SD[Metric4-Bi]/SDBi & 1.4924 & 0.6638 & 1.1733 & 1.4332 & 0.2562 & 0.6273 & 1.2728 & 1.1156 & 1.2503 & 1.3844 \\
\hline Mean[Metric5] & 0.0550 & 0.0112 & 0.0191 & 0.0550 & 0.0258 & 0.0310 & 0.0610 & 0.0139 & 0.0217 & 0.0570 \\
\hline SD[Metric5] & 0.0011 & 0.0013 & 0.0010 & 0.0013 & 0.0044 & 0.0043 & 0.0030 & 0.0005 & 0.0007 & 0.0014 \\
\hline Mean[Metric5-Bi] & 0.0151 & 0.0003 & 0.0031 & 0.0144 & -0.0002 & 0.0013 & 0.0127 & -0.0005 & 0.0007 & 0.0114 \\
\hline SD[Metric5-Bi] & 0.0057 & 0.0010 & 0.0022 & 0.0059 & 0.0012 & 0.0028 & 0.0071 & 0.0008 & 0.0020 & 0.0061 \\
\hline Mean[ Metric5-Bi/Bi] & 0.4063 & 0.0316 & 0.2098 & 0.3805 & -0.0074 & 0.0538 & 0.2865 & -0.0298 & 0.0433 & 0.2712 \\
\hline SD[ Metric5-Bi/Bi] & 0.1948 & 0.0908 & 0.1596 & 0.1912 & 0.0458 & 0.1002 & 0.1824 & 0.0540 & 0.0969 & 0.1646 \\
\hline SD[Metric5]/SDBi & 0.1999 & 0.8584 & 0.4735 & 0.2267 & 0.9686 & 0.8788 & 0.4360 & 0.7115 & 0.3887 & 0.2342 \\
\hline SD[Metric5-Bi]/SDBi & 1.0285 & 0.6366 & 1.0033 & 1.0097 & 0.2551 & 0.5866 & 1.0231 & 1.0700 & 1.0569 & 1.0229 \\
\hline Mean[Metric6] & 0.0586 & 0.0122 & 0.0212 & 0.0591 & 0.0267 & 0.0333 & 0.0670 & 0.0149 & 0.0239 & 0.0618 \\
\hline SD[Metric6] & 0.0011 & 0.0013 & 0.0011 & 0.0014 & 0.0045 & 0.0045 & 0.0035 & 0.0005 & 0.0006 & 0.0013 \\
\hline Mean[Metric6-Bi] & 0.0188 & 0.0013 & 0.0051 & 0.0184 & 0.0006 & 0.0036 & 0.0186 & 0.0005 & 0.0029 & 0.0162 \\
\hline SD[Metric6-Bi] & 0.0057 & 0.0009 & 0.0022 & 0.0059 & 0.0014 & 0.0031 & 0.0071 & 0.0008 & 0.0020 & 0.0061 \\
\hline Mean[ Metric6-Bi/Bi] & 0.5007 & 0.1261 & 0.3391 & 0.4823 & 0.0253 & 0.1312 & 0.4115 & 0.0405 & 0.1463 & 0.3781 \\
\hline SD[ Metric6-Bi/Bi] & 0.2065 & 0.0950 & 0.1725 & 0.2050 & 0.0553 & 0.1143 & 0.1981 & 0.0548 & 0.1044 & 0.1782 \\
\hline SD[Metric6]/SDBi & 0.2031 & 0.8682 & 0.5183 & 0.2346 & 0.9925 & 0.9306 & 0.5094 & 0.6809 & 0.3395 & 0.2112 \\
\hline SD[Metric6-Bi]/SDBi & 1.0220 & 0.6133 & 0.9880 & 1.0108 & 0.3020 & 0.6451 & 1.0245 & 1.0320 & 1.0454 & 1.0254 \\
\hline Mean[Metric7] & 0.0569 & 0.0143 & 0.0231 & 0.0586 & 0.0309 & 0.0395 & 0.0726 & 0.0166 & 0.0259 & 0.0608 \\
\hline SD[Metric7] & 0.0084 & 0.0017 & 0.0031 & 0.0084 & 0.0045 & 0.0054 & 0.0094 & 0.0011 & 0.0030 & 0.0096 \\
\hline Mean[Metric7-Bi] & 0.0171 & 0.0034 & 0.0070 & 0.0180 & 0.0048 & 0.0097 & 0.0242 & 0.0023 & 0.0050 & 0.0152 \\
\hline SD[Metric7-Bi] & 0.0100 & 0.0018 & 0.0037 & 0.0102 & 0.0029 & 0.0045 & 0.0109 & 0.0012 & 0.0036 & 0.0110 \\
\hline Mean[ Metric7-Bi/Bi] & 0.4560 & 0.3266 & 0.4595 & 0.4704 & 0.1963 & 0.3452 & 0.5282 & 0.1599 & 0.2466 & 0.3547 \\
\hline SD[ Metric7-Bi/Bi] & 0.2930 & 0.2015 & 0.2671 & 0.2916 & 0.1323 & 0.1813 & 0.2736 & 0.0905 & 0.1828 & 0.2680 \\
\hline SD[Metric7]/SDBi & 1.5021 & 1.0966 & 1.4195 & 1.4301 & 0.9903 & 1.1070 & 1.3558 & 1.4385 & 1.5647 & 1.6040 \\
\hline SD[Metric7-Bi]/SDBi & 1.7900 & 1.1806 & 1.6824 & 1.7406 & 0.6315 & 0.9333 & 1.5716 & 1.6435 & 1.8826 & 1.8426 \\
\hline Mean[Metric8] & 0.0812 & 0.0170 & 0.0308 & 0.0827 & 0.0336 & 0.0473 & 0.0972 & 0.0194 & 0.0340 & 0.0876 \\
\hline SD[Metric8] & 0.0021 & 0.0013 & 0.0014 & 0.0025 & 0.0044 & 0.0046 & 0.0049 & 0.0005 & 0.0007 & 0.0020 \\
\hline Mean[Metric8-Bi] & 0.0414 & 0.0061 & 0.0148 & 0.0420 & 0.0075 & 0.0176 & 0.0489 & 0.0050 & 0.0130 & 0.0420 \\
\hline SD[Metric8-Bi] & 0.0059 & 0.0015 & 0.0024 & 0.0062 & 0.0027 & 0.0035 & 0.0076 & 0.0008 & 0.0020 & 0.0063 \\
\hline Mean[ Metric8-Bi/Bi] & 1.0777 & 0.5772 & 0.9510 & 1.0741 & 0.3048 & 0.6167 & 1.0491 & 0.3520 & 0.6324 & 0.9518 \\
\hline SD[ Metric8-Bi/Bi] & 0.2869 & 0.2009 & 0.2618 & 0.2892 & 0.1375 & 0.1881 & 0.2863 & 0.0715 & 0.1484 & 0.2525 \\
\hline SD[Metric8]/SDBi & 0.3823 & 0.8712 & 0.6580 & 0.4185 & 0.9545 & 0.9421 & 0.7022 & 0.6588 & 0.3922 & 0.3362 \\
\hline SD[Metric8-Bi]/SDBi & 1.0680 & 0.9600 & 1.1023 & 1.0609 & 0.5842 & 0.7333 & 1.0998 & 1.0835 & 1.0811 & 1.0569 \\
\hline Mean[Metric9] & 0.0559 & 0.0135 & 0.0223 & 0.0572 & 0.0289 & 0.0377 & 0.0703 & 0.0163 & 0.0255 & 0.0596 \\
\hline SD[Metric9] & 0.0084 & 0.0017 & 0.0030 & 0.0083 & 0.0046 & 0.0054 & 0.0093 & 0.0011 & 0.0030 & 0.0096 \\
\hline Mean[Metric9-Bi] & 0.0160 & 0.0025 & 0.0062 & 0.0166 & 0.0028 & 0.0080 & 0.0220 & 0.0020 & 0.0045 & 0.0140 \\
\hline SD[Metric9-Bi] & 0.0100 & 0.0014 & 0.0035 & 0.0102 & 0.0015 & 0.0041 & 0.0107 & 0.0012 & 0.0035 & 0.0110 \\
\hline Mean[ Metric9-Bi/Bi] & 0.4291 & 0.2405 & 0.4059 & 0.4357 & 0.1115 & 0.2819 & 0.4803 & 0.1400 & 0.2254 & 0.3277 \\
\hline SD[ Metric9-Bi/Bi] & 0.2917 & 0.1413 & 0.2502 & 0.2875 & 0.0641 & 0.1565 & 0.2658 & 0.0880 & 0.1806 & 0.2669 \\
\hline SD[Metric9]/SDBi & 1.5052 & 1.1358 & 1.3940 & 1.4134 & 1.0112 & 1.1253 & 1.3414 & 1.4648 & 1.5657 & 1.6055 \\
\hline SD[Metric9-Bi]/SDBi & 1.7982 & 0.9023 & 1.6064 & 1.7362 & 0.3210 & 0.8478 & 1.5453 & 1.6168 & 1.8717 & 1.8466 \\
\hline formMean & 0.0369 & 0.0037 & 0.0110 & 0.0367 & 0.0037 & 0.0110 & 0.0369 & 0.0037 & 0.0110 & 0.0364 \\
\hline formStdDev & 0.0070 & 0.0007 & 0.0020 & 0.0068 & 0.0007 & 0.0021 & 0.0069 & 0.0007 & 0.0020 & 0.0068 \\
\hline Points per sphere & 25 & 25 & 25 & 25 & 25 & 25 & 25 & 25 & 25 & 25 \\
\hline Scenario number & 12 & 13 & 14 & 15 & 16 & 17 & 18 & 19 & 20 & 21 \\
\hline
\end{tabular}


13

\begin{tabular}{|c|c|c|c|c|c|c|c|c|c|c|}
\hline Scenario number & 22 & 23 & 24 & 25 & 26 & 27 & 28 & 29 & 30 & 31 \\
\hline IongRangeErrorDist & $\mathrm{N}[0 ., 0.003]$ & $\mathrm{N}[0 ., 0.003]$ & $\mathrm{N}[0 ., 0.003]$ & $\mathrm{N}[0 ., 0.010]$ & $\mathrm{N}[0 ., 0.010]$ & $\mathrm{N}[0 ., 0.010]$ & $\mathrm{N}[0.003,0.001]$ & $\mathrm{N}[0.003,0.001]$ & $\mathrm{N}[0.003,0.001]$ & $\mathrm{N}[0.003,0.003]$ \\
\hline probeRndErrorDist & $\mathrm{N}[0.005,0.001]$ & $\mathrm{N}[0.005,0.003]$ & $\mathrm{N}[0.005,0.010]$ & $\mathrm{N}[0.005,0.001]$ & $\mathrm{N}[0.005,0.003]$ & $\mathrm{N}[0.005,0.010]$ & $\mathrm{N}[0 ., 0.001]$ & $\mathrm{N}[0 ., 0.003]$ & $\mathrm{N}[0 ., 0.010]$ & $N[0 ., 0.001]$ \\
\hline Mean[Bi] & 0.0180 & 0.0231 & 0.0462 & 0.0323 & 0.0357 & 0.0539 & 0.0073 & 0.0141 & 0.0401 & 0.0109 \\
\hline stdDevBi & 0.0016 & 0.0023 & 0.0062 & 0.0049 & 0.0051 & 0.0075 & 0.0007 & 0.0019 & 0.0058 & 0.0016 \\
\hline Mean[Metric1] & 0.0185 & 0.0244 & 0.0498 & 0.0345 & 0.0375 & 0.0575 & 0.0068 & 0.0134 & 0.0398 & 0.0105 \\
\hline SD[Metric1] & 0.0014 & 0.0026 & 0.0090 & 0.0044 & 0.0046 & 0.0093 & 0.0009 & 0.0027 & 0.0091 & 0.0016 \\
\hline Mean[Metric1-Bi] & 0.0005 & 0.0013 & 0.0036 & 0.0022 & 0.0018 & 0.0036 & -0.0005 & -0.0007 & -0.0003 & -0.0004 \\
\hline SD[Metric1-Bi] & 0.0015 & 0.0034 & 0.0108 & 0.0042 & 0.0050 & 0.0116 & 0.0011 & 0.0033 & 0.0106 & 0.0012 \\
\hline Mean[ Metric1-Bi/Bi] & 0.0333 & 0.0680 & 0.0953 & 0.0797 & 0.0635 & 0.0845 & -0.0625 & -0.0302 & 0.0102 & -0.0312 \\
\hline SD[ Metric1-Bi/Bi] & 0.0878 & 0.1511 & 0.2399 & 0.1534 & 0.1509 & 0.2220 & 0.1451 & 0.2358 & 0.2682 & 0.1085 \\
\hline SD[Metric1]/SDBi & 0.8847 & 1.1132 & 1.4539 & 0.8967 & 0.9080 & 1.2399 & 1.1843 & 1.3947 & 1.5673 & 1.0119 \\
\hline SD[Metric1-Bi]/SDBi & 0.9653 & 1.4744 & 1.7475 & 0.8610 & 0.9768 & 1.5436 & 1.4579 & 1.7525 & 1.8110 & 0.7612 \\
\hline Mean[Metric2] & 0.0204 & 0.0315 & 0.0749 & 0.0360 & 0.0434 & 0.0821 & 0.0090 & 0.0209 & 0.0663 & 0.0124 \\
\hline SD[Metric2] & 0.0011 & 0.0007 & 0.0008 & 0.0043 & 0.0037 & 0.0025 & 0.0004 & 0.0004 & 0.0006 & 0.0013 \\
\hline Mean[Metric2-Bi] & 0.0025 & 0.0084 & 0.0286 & 0.0037 & 0.0077 & 0.0282 & 0.0017 & 0.0068 & 0.0261 & 0.0015 \\
\hline SD[Metric2-Bi] & 0.0014 & 0.0023 & 0.0063 & 0.0041 & 0.0044 & 0.0077 & 0.0008 & 0.0019 & 0.0059 & 0.0010 \\
\hline Mean[ Metric2-Bi/Bi] & 0.1433 & 0.3770 & 0.6479 & 0.1274 & 0.2318 & 0.5503 & 0.2385 & 0.5085 & 0.6843 & 0.1445 \\
\hline SD[ Metric2-Bi/Bi] & 0.0870 & 0.1343 & 0.2156 & 0.1561 & 0.1544 & 0.2089 & 0.1227 & 0.1995 & 0.2367 & 0.1001 \\
\hline SD[Metric2]/SDBi & 0.7174 & 0.3121 & 0.1233 & 0.8799 & 0.7353 & 0.3291 & 0.5420 & 0.1924 & 0.1085 & 0.8471 \\
\hline SD[Metric2-Bi]/SDBi & 0.8742 & 1.0120 & 1.0085 & 0.8483 & 0.8706 & 1.0167 & 1.0329 & 1.0205 & 1.0039 & 0.6099 \\
\hline Mean[Metric3] & 0.0172 & 0.0214 & 0.0445 & 0.0317 & 0.0343 & 0.0529 & 0.0068 & 0.0136 & 0.0414 & 0.0105 \\
\hline SD[Metric3] & 0.0016 & 0.0023 & 0.0067 & 0.0048 & 0.0050 & 0.0076 & 0.0008 & 0.0020 & 0.0065 & 0.0016 \\
\hline Mean[Metric3-Bi] & \begin{tabular}{|l|} 
\\
\end{tabular} & -0.0017 & -0.0018 & -0.0006 & -0.0014 & -0.0011 & -0.0005 & -0.0005 & 0.0013 & -0.0004 \\
\hline SD[Metric3-Bi] & 0.0012 & 0.0031 & 0.0091 & 0.0012 & 0.0036 & 0.0101 & 0.0010 & 0.0028 & 0.0086 & 0.0012 \\
\hline Mean[ Metric3-Bi/Bi] & -0.0393 & -0.0660 & -0.0221 & -0.0190 & -0.0354 & -0.0033 & -0.0607 & -0.0186 & 0.0526 & -0.0305 \\
\hline SD[ Metric3-Bi/Bi] & 0.0650 & 0.1272 & 0.1916 & 0.0385 & 0.0987 & 0.1859 & 0.1359 & 0.1992 & 0.2180 & 0.1058 \\
\hline SD[Metric3]/SDBi & 1.0306 & 0.9928 & 1.0818 & 0.9834 & 0.9842 & 1.0159 & 1.0758 & 1.0573 & 1.1130 & 0.9937 \\
\hline SD[Metric3-Bi]/SDBi & 0.7524 & 1.3236 & 1.4627 & 0.2534 & 0.6988 & 1.3486 & 1.3731 & 1.4921 & 1.4702 & 0.7445 \\
\hline Mean[Metric4] & 0.0174 & 0.0220 & 0.0464 & 0.0319 & 0.0349 & 0.0543 & 0.0071 & 0.0142 & 0.0422 & 0.0107 \\
\hline SD[Metric4] & 0.0015 & 0.0018 & 0.0055 & 0.0048 & 0.0046 & 0.0063 & 0.0006 & 0.0017 & 0.0062 & 0.0014 \\
\hline Mean[Metric4-Bi] & -0.0005 & -0.0011 & 0.0002 & -0.0005 & -0.0008 & 0.0004 & -0.0002 & 0.0001 & 0.0020 & -0.0002 \\
\hline SD[Metric4-Bi] & 0.0010 & 0.0027 & 0.0083 & 0.0011 & 0.0031 & 0.0093 & 0.0009 & 0.0026 & 0.0083 & 0.0010 \\
\hline Mean[ Metric4-Bi/Bi] & -0.0275 & -0.0401 & 0.0218 & -0.0134 & -0.0186 & 0.0241 & -0.0258 & 0.0241 & 0.0709 & -0.0148 \\
\hline SD[ Metric4-Bi/Bi] & 0.0558 & 0.1109 & 0.1785 & 0.0350 & 0.0871 & 0.1726 & 0.1168 & 0.1830 & 0.2139 & 0.0946 \\
\hline SD[Metric4]/SDBi & 0.9299 & 0.7665 & 0.8922 & 0.9783 & 0.9077 & 0.8330 & 0.8068 & 0.8755 & 1.0599 & 0.9050 \\
\hline SD[Metric4-Bi]/SDBi & 0.6491 & 1.1542 & 1.3347 & 0.2323 & 0.6122 & 1.2363 & 1.1814 & 1.3483 & 1.4302 & 0.6653 \\
\hline Mean[Metric5] & 0.0178 & 0.0242 & 0.0571 & 0.0321 & 0.0364 & 0.0643 & 0.0078 & 0.0174 & 0.0544 & 0.0112 \\
\hline SD[Metric5] & 0.0014 & 0.0013 & 0.0016 & 0.0047 & 0.0044 & 0.0034 & 0.0004 & 0.0005 & 0.0010 & 0.0013 \\
\hline Mean[Metric5-Bi] & -0.0002 & 0.0011 & 0.0109 & -0.0003 & 0.0007 & 0.0104 & 0.0005 & 0.0033 & 0.0143 & 0.0003 \\
\hline SD[Metric5-Bi] & 0.0010 & 0.0024 & 0.0063 & 0.0011 & 0.0030 & 0.0076 & 0.0008 & 0.0020 & 0.0059 & 0.0010 \\
\hline Mean[ Metric5-Bi/Bi] & -0.0094 & 0.0584 & 0.2573 & -0.0074 & 0.0249 & 0.2138 & 0.0817 & 0.2574 & 0.3828 & 0.0349 \\
\hline SD[ Metric5-Bi/Bi] & 0.0559 & 0.1071 & 0.1660 & 0.0349 & 0.0871 & 0.1619 & 0.1122 & 0.1680 & 0.1948 & 0.0946 \\
\hline SD[Metric5]/SDBi & 0.9099 & 0.5746 & 0.2608 & 0.9719 & 0.8615 & 0.4472 & 0.5927 & 0.2729 & 0.1793 & 0.8544 \\
\hline SD[Metric5-Bi]/SDBi & 0.6451 & 1.0469 & 1.0215 & 0.2309 & 0.5948 & 1.0042 & 1.0619 & 1.0295 & 1.0115 & 0.6396 \\
\hline Mean[Metric6] & 0.0188 & 0.0267 & 0.0621 & 0.0331 & 0.0394 & 0.0711 & 0.0087 & 0.0190 & 0.0583 & 0.0123 \\
\hline SD[Metric6] & 0.0014 & 0.0013 & 0.0016 & 0.0047 & 0.0045 & 0.0036 & 0.0004 & 0.0005 & 0.0011 & 0.0014 \\
\hline Mean[Metric6-Bi] & 0.0008 & 0.0036 & 0.0158 & 0.0008 & 0.0037 & 0.0172 & 0.0014 & 0.0049 & 0.0181 & 0.0013 \\
\hline SD[Metric6-Bi] & 0.0010 & 0.0024 & 0.0064 & 0.0011 & 0.0030 & 0.0076 & 0.0008 & 0.0020 & 0.0059 & 0.0010 \\
\hline Mean[ Metric6-Bi/Bi] & 0.0494 & 0.1648 & 0.3661 & 0.0260 & 0.1117 & 0.3420 & 0.1975 & 0.3719 & 0.4811 & 0.1311 \\
\hline SD[ Metric6-Bi/Bi] & 0.0565 & 0.1141 & 0.1799 & 0.0347 & 0.0936 & 0.1771 & 0.1204 & 0.1834 & 0.2091 & 0.0987 \\
\hline SD[Metric6]/SDBi & 0.9106 & 0.5403 & 0.2523 & 0.9749 & 0.8766 & 0.4745 & 0.5870 & 0.2584 & 0.1803 & 0.8679 \\
\hline SD[Metric6-Bi]/SDBi & 0.6240 & 1.0237 & 1.0251 & 0.2202 & 0.5900 & 1.0037 & 1.0482 & 1.0358 & 1.0128 & 0.6130 \\
\hline Mean[Metric7] & 0.0213 & 0.0301 & 0.0626 & 0.0379 & 0.0461 & 0.0767 & 0.0099 & 0.0202 & 0.0578 & 0.0140 \\
\hline SD[Metric7] & 0.0016 & 0.0031 & 0.0093 & 0.0045 & 0.0053 & 0.0106 & 0.0009 & 0.0024 & 0.0081 & 0.0017 \\
\hline Mean[Metric7-Bi] & 0.0033 & 0.0070 & 0.0163 & 0.0055 & 0.0104 & 0.0228 & 0.0026 & 0.0061 & 0.0177 & 0.0031 \\
\hline SD[Metric7-Bi] & 0.0017 & 0.0038 & 0.0111 & 0.0042 & 0.0054 & 0.0126 & 0.0011 & 0.0031 & 0.0099 & 0.0013 \\
\hline Mean[ Metric7-Bi/Bi] & 0.1896 & 0.3157 & 0.3763 & 0.1862 & 0.3079 & 0.4481 & 0.3688 & 0.4599 & 0.4687 & 0.2939 \\
\hline SD[ Metric7-Bi/Bi] & 0.1025 & 0.1813 & 0.2679 & 0.1646 & 0.1839 & 0.2696 & 0.1767 & 0.2625 & 0.2881 & 0.1356 \\
\hline SD[Metric7]/SDBi & 1.0257 & 1.3183 & 1.5011 & 0.9169 & 1.0368 & 1.4024 & 1.2525 & 1.2574 & 1.3803 & 1.0764 \\
\hline SD[Metric7-Bi]/SDBi & 1.0449 & 1.6211 & 1.7903 & 0.8699 & 1.0594 & 1.6722 & 1.5154 & 1.6375 & 1.6900 & 0.8114 \\
\hline Mean[Metric8] & 0.0239 & 0.0379 & 0.0883 & 0.0405 & 0.0543 & 0.1035 & 0.0121 & 0.0266 & 0.0796 & 0.0162 \\
\hline SD[Metric8] & 0.0013 & 0.0014 & 0.0024 & 0.0044 & 0.0043 & 0.0047 & 0.0005 & 0.0008 & 0.0021 & 0.0015 \\
\hline Mean[Metric8-Bi] & 0.0060 & 0.0148 & 0.0421 & 0.0082 & 0.0186 & 0.0496 & 0.0048 & 0.0125 & 0.0395 & 0.0053 \\
\hline SD[Metric8-Bi] & 0.0014 & 0.0026 & 0.0066 & 0.0042 & 0.0046 & 0.0084 & 0.0008 & 0.0021 & 0.0062 & 0.0010 \\
\hline Mean[ Metric8-Bi/Bi] & 0.3383 & 0.6585 & 0.9435 & 0.2697 & 0.5412 & 0.9550 & 0.6699 & 0.9235 & 1.0245 & 0.4973 \\
\hline SD[ Metric8-Bi/Bi] & 0.1020 & 0.1658 & 0.2589 & 0.1716 & 0.1917 & 0.2673 & 0.1652 & 0.2588 & 0.2881 & 0.1345 \\
\hline SD[Metric8]/SDBi & 0.8518 & 0.5983 & 0.3802 & 0.8986 & 0.8527 & 0.6288 & 0.7186 & 0.4170 & 0.3517 & 0.9482 \\
\hline SD[Metric8-Bi]/SDBi & 0.9122 & 1.1016 & 1.0715 & 0.8545 & 0.9042 & 1.1161 & 1.1021 & 1.0882 & 1.0549 & 0.6277 \\
\hline Mean[Metric9] & 0.0205 & 0.0292 & 0.0613 & 0.0351 & 0.0434 & 0.0741 & 0.0094 & 0.0187 & 0.0553 & 0.0135 \\
\hline SD[Metric9] & 0.0018 & 0.0031 & 0.0094 & 0.0049 & 0.0057 & 0.0106 & 0.0011 & 0.0028 & 0.0083 & 0.0018 \\
\hline Mean[Metric9-Bi] & 0.0025 & 0.0061 & 0.0150 & 0.0028 & 0.0077 & 0.0202 & 0.0021 & 0.0046 & 0.0151 & 0.0026 \\
\hline SD[Metric9-Bi] & 0.0013 & 0.0037 & 0.0111 & 0.0014 & 0.0041 & 0.0124 & 0.0012 & 0.0035 & 0.0099 & 0.0014 \\
\hline Mean[ Metric9-Bi/Bi] & 0.1433 & 0.2766 & 0.3475 & 0.0897 & 0.2231 & 0.3971 & 0.2919 & 0.3517 & 0.4035 & 0.2468 \\
\hline SD[ Metric9-Bi/Bi] & 0.0784 & 0.1746 & 0.2650 & 0.0486 & 0.1294 & 0.2606 & 0.1860 & 0.2765 & 0.2826 & 0.1406 \\
\hline SD[Metric9]/SDBi & 1.1267 & 1.3383 & 1.5093 & 1.0050 & 1.1096 & 1.4151 & 1.4539 & 1.4781 & 1.4169 & 1.1335 \\
\hline SD[Metric9-Bi]/SDBi & 0.8426 & 1.5904 & 1.7930 & 0.2919 & 0.8119 & 1.6486 & 1.6652 & 1.8255 & 1.7045 & 0.8779 \\
\hline formMean & 0.0037 & 0.0110 & 0.0361 & 0.0037 & 0.0110 & 0.0368 & 0.0037 & 0.0110 & 0.0367 & 0.0037 \\
\hline formStdDev & 0.0007 & 0.0020 & 0.0066 & 0.0007 & 0.0021 & 0.0069 & 0.0007 & 0.0020 & 0.0068 & 0.0007 \\
\hline Points per sphere & 25 & 25 & 25 & 25 & 25 & 25 & 25 & 25 & 25 & 25 \\
\hline Scenario number & 22 & 23 & 24 & 25 & 26 & 27 & 28 & 29 & 30 & 31 \\
\hline
\end{tabular}


14

\begin{tabular}{|c|c|c|c|c|c|c|c|c|c|c|}
\hline Scenario number & 32 & 33 & 34 & 35 & 36 & 37 & 38 & 39 & 40 & 41 \\
\hline IongRangeErrorDist & $\mathrm{N}[0.003,0.003]$ & $\mathrm{N}[0.003,0.003]$ & $\mathrm{N}[0.003,0.010]$ & $\mathrm{N}[0.003,0.010]$ & $\mathrm{N}[0.003,0.010]$ & $\mathrm{N}[0.003,0.001]$ & $\mathrm{N}[0.003,0.001]$ & $\mathrm{N}[0.003,0.001]$ & $\mathrm{N}[0.003,0.003]$ & $\mathrm{N}[0.003,0.003]$ \\
\hline probeRndErrorDist & $\mathrm{N}[0 ., 0.003]$ & $\mathrm{N}[0 ., 0.010]$ & $\mathrm{N}[0 ., 0.001]$ & $\mathrm{N}[0 ., 0.003]$ & $\mathrm{N}[0 ., 0.010]$ & $\mathrm{N}[0.0015,0.001]$ & $\mathrm{N}[0.0015,0.003]$ & $\mathrm{N}[0.0015,0.010]$ & $\mathrm{N}[0.0015,0.001]$ & $\mathrm{N}[0.0015,0.003]$ \\
\hline Mean[Bi] & 0.0161 & 0.0412 & 0.0261 & 0.0295 & 0.0486 & 0.0104 & 0.0170 & 0.0422 & 0.0139 & 0.0190 \\
\hline stdDevBi & 0.0023 & 0.0061 & 0.0044 & 0.0051 & 0.0073 & 0.0008 & 0.0019 & 0.0058 & 0.0016 & 0.0023 \\
\hline Mean[Metric1] & 0.0158 & 0.0409 & 0.0256 & 0.0286 & 0.0485 & 0.0098 & 0.0164 & 0.0429 & 0.0135 & 0.0186 \\
\hline SD[Metric1] & 0.0026 & 0.0088 & 0.0045 & 0.0050 & 0.0092 & 0.0009 & 0.0027 & 0.0090 & 0.0016 & 0.0027 \\
\hline Mean[Metric1-Bi] & -0.0004 & -0.0003 & -0.0006 & -0.0009 & -0.0001 & -0.0005 & -0.0006 & 0.0007 & -0.0004 & -0.0004 \\
\hline SD[Metric1-Bi] & 0.0033 & 0.0104 & 0.0012 & 0.0036 & 0.0113 & 0.0011 & 0.0032 & 0.0108 & 0.0012 & 0.0033 \\
\hline Mean[ Metric1-Bi/Bi] & -0.0060 & 0.0122 & -0.0216 & -0.0241 & 0.0168 & -0.0470 & -0.0245 & 0.0368 & -0.0266 & -0.0105 \\
\hline SD[ Metric1-Bi/Bi] & 0.2041 & 0.2553 & 0.0480 & 0.1252 & 0.2332 & 0.1037 & 0.1857 & 0.2625 & 0.0829 & 0.1743 \\
\hline SD[Metric1]/SDBi & 1.1632 & 1.4373 & 1.0038 & 0.9761 & 1.2701 & 1.1109 & 1.4284 & 1.5314 & 0.9938 & 1.1943 \\
\hline SD[Metric1-Bi]/SDBi & 1.4645 & 1.7048 & 0.2734 & 0.7138 & 1.5529 & 1.3769 & 1.7077 & 1.8431 & 0.7420 & 1.4542 \\
\hline Mean[Metric2] & 0.0226 & 0.0662 & 0.0272 & 0.0346 & 0.0730 & 0.0120 & 0.0238 & 0.0687 & 0.0153 & 0.0257 \\
\hline SD[Metric2] & 0.0009 & 0.0009 & 0.0044 & 0.0041 & 0.0026 & 0.0004 & 0.0004 & 0.0006 & 0.0013 & 0.0009 \\
\hline Mean[Metric2-Bi] & 0.0065 & 0.0250 & 0.0010 & 0.0051 & 0.0245 & 0.0016 & 0.0068 & 0.0265 & 0.0014 & 0.0067 \\
\hline SD[Metric2-Bi] & 0.0022 & 0.0062 & 0.0010 & 0.0029 & 0.0071 & 0.0008 & 0.0019 & 0.0059 & 0.0009 & 0.0022 \\
\hline Mean[ Metric2-Bi/Bi] & 0.4272 & 0.6423 & 0.0418 & 0.1867 & 0.5334 & 0.1603 & 0.4130 & 0.6592 & 0.1082 & 0.3686 \\
\hline SD[ Metric2-Bi/Bi] & 0.1848 & 0.2346 & 0.0418 & 0.1196 & 0.2120 & 0.0853 & 0.1520 & 0.2283 & 0.0748 & 0.1527 \\
\hline SD[Metric2]/SDBi & 0.4154 & 0.1448 & 0.9876 & 0.8045 & 0.3609 & 0.5060 & 0.1941 & 0.1072 & 0.8549 & 0.4148 \\
\hline SD[Metric2-Bi]/SDBi & 0.9701 & 1.0081 & 0.2281 & 0.5732 & 0.9721 & 0.9840 & 1.0102 & 1.0078 & 0.6042 & 0.9693 \\
\hline Mean[Metric3] & 0.0159 & 0.0422 & 0.0256 & 0.0288 & 0.0502 & 0.0095 & 0.0154 & 0.0421 & 0.0133 & 0.0179 \\
\hline SD[Metric3] & 0.0022 & 0.0062 & 0.0044 & 0.0046 & 0.0068 & 0.0008 & 0.0021 & 0.0066 & 0.0016 & 0.0024 \\
\hline Mean[Metric3-Bi] & -0.0002 & 0.0011 & -0.0005 & -0.0008 & 0.0016 & -0.0009 & -0.0016 & -0.0001 & -0.0006 & -0.0011 \\
\hline SD[Metric3-Bi] & 0.0030 & 0.0085 & 0.0012 & 0.0034 & 0.0093 & 0.0011 & 0.0027 & 0.0088 & 0.0011 & 0.0030 \\
\hline Mean[ Metric3-Bi/Bi] & 0.0025 & 0.0466 & -0.0199 & -0.0172 & 0.0527 & -0.0806 & -0.0866 & 0.0162 & -0.0385 & -0.0473 \\
\hline SD[ Metric3-Bi/Bi] & 0.1838 & 0.2109 & 0.0478 & 0.1171 & 0.1938 & 0.0968 & 0.1547 & 0.2139 & 0.0811 & 0.1555 \\
\hline SD[Metric3]/SDBi & 0.9664 & 1.0078 & 0.9962 & 0.9139 & 0.9349 & 1.0389 & 1.1266 & 1.1332 & 1.0058 & 1.0389 \\
\hline SD[Metric3-Bi]/SDBi & 1.3236 & 1.3939 & 0.2740 & 0.6764 & 1.2841 & 1.3092 & 1.4695 & 1.5102 & 0.7294 & 1.3261 \\
\hline Mean[Metric4] & 0.0162 & 0.0427 & 0.0257 & 0.0290 & 0.0504 & 0.0097 & 0.0162 & 0.0435 & 0.0135 & 0.0186 \\
\hline SD[Metric4] & 0.0018 & 0.0059 & 0.0044 & 0.0045 & 0.0064 & 0.0006 & 0.0016 & 0.0061 & 0.0015 & 0.0019 \\
\hline Mean[Metric4-Bi] & 0.0001 & 0.0016 & -0.0004 & -0.0005 & 0.0018 & -0.0007 & -0.0009 & 0.0013 & -0.0004 & -0.0005 \\
\hline SD[Metric4-Bi] & 0.0027 & 0.0083 & 0.0011 & 0.0032 & 0.0090 & 0.0009 & 0.0023 & 0.0084 & 0.0010 & 0.0027 \\
\hline Mean[ Metric4-Bi/Bi] & 0.0247 & 0.0593 & -0.0146 & -0.0089 & 0.0566 & -0.0584 & -0.0409 & 0.0502 & -0.0235 & -0.0125 \\
\hline SD[ Metric4-Bi/Bi] & 0.1714 & 0.2068 & 0.0413 & 0.1094 & 0.1868 & 0.0801 & 0.1333 & 0.2072 & 0.0724 & 0.1415 \\
\hline SD[Metric4]/SDBi & 0.8192 & 0.9656 & 0.9961 & 0.8892 & 0.8859 & 0.7304 & 0.8391 & 1.0422 & 0.9429 & 0.8198 \\
\hline SD[Metric4-Bi]/SDBi & 1.2159 & 1.3613 & 0.2375 & 0.6342 & 1.2355 & 1.0910 & 1.2582 & 1.4373 & 0.6555 & 1.2025 \\
\hline Mean[Metric5] & 0.0193 & 0.0544 & 0.0260 & 0.0310 & 0.0613 & 0.0103 & 0.0191 & 0.0554 & 0.0139 & 0.0212 \\
\hline SD[Metric5] & 0.0010 & 0.0013 & 0.0044 & 0.0041 & 0.0030 & 0.0005 & 0.0006 & 0.0012 & 0.0014 & 0.0012 \\
\hline Mean[Metric5-Bi] & 0.0031 & 0.0132 & -0.0002 & 0.0014 & 0.0127 & -0.0001 & 0.0020 & 0.0132 & 0.0000 & 0.0022 \\
\hline SD[Metric5-Bi] & 0.0022 & 0.0062 & 0.0010 & 0.0031 & 0.0073 & 0.0008 & 0.0019 & 0.0059 & 0.0010 & 0.0023 \\
\hline Mean[ Metric5-Bi/Bi] & 0.2144 & 0.3485 & -0.0057 & 0.0600 & 0.2870 & -0.0016 & 0.1320 & 0.3382 & 0.0073 & 0.1275 \\
\hline SD[ Metric5-Bi/Bi] & 0.1617 & 0.1930 & 0.0411 & 0.1101 & 0.1827 & 0.0778 & 0.1251 & 0.1857 & 0.0719 & 0.1298 \\
\hline SD[Metric5]/SDBi & 0.4592 & 0.2203 & 0.9875 & 0.8139 & 0.4202 & 0.6093 & 0.3467 & 0.2132 & 0.9076 & 0.5314 \\
\hline SD[Metric5-Bi]/SDBi & 0.9967 & 1.0170 & 0.2359 & 0.6049 & 1.0050 & 1.0255 & 1.0432 & 1.0176 & 0.6375 & 1.0082 \\
\hline Mean[Metric6] & 0.0213 & 0.0586 & 0.0268 & 0.0335 & 0.0669 & 0.0112 & 0.0209 & 0.0595 & 0.0150 & 0.0235 \\
\hline SD[Metric6] & 0.0011 & 0.0014 & 0.0045 & 0.0044 & 0.0034 & 0.0005 & 0.0005 & 0.0011 & 0.0014 & 0.0012 \\
\hline Mean[Metric6-Bi] & 0.0051 & 0.0174 & 0.0006 & 0.0040 & 0.0183 & 0.0009 & 0.0039 & 0.0173 & 0.0011 & 0.0045 \\
\hline SD[Metric6-Bi] & 0.0022 & 0.0062 & 0.0013 & 0.0032 & 0.0072 & 0.0008 & 0.0019 & 0.0060 & 0.0010 & 0.0022 \\
\hline Mean[ Metric6-Bi/Bi] & 0.3402 & 0.4536 & 0.0252 & 0.1474 & 0.4044 & 0.0896 & 0.2406 & 0.4362 & 0.0815 & 0.2518 \\
\hline SD[ Metric6-Bi/Bi] & 0.1745 & 0.2093 & 0.0526 & 0.1240 & 0.1967 & 0.0823 & 0.1353 & 0.1994 & 0.0729 & 0.1413 \\
\hline SD[Metric6]/SDBi & 0.4904 & 0.2253 & 1.0128 & 0.8686 & 0.4732 & 0.6010 & 0.2949 & 0.1892 & 0.9058 & 0.5310 \\
\hline SD[Metric6-Bi]/SDBi & 0.9831 & 1.0211 & 0.2998 & 0.6391 & 0.9972 & 1.0093 & 1.0301 & 1.0212 & 0.6095 & 0.9906 \\
\hline Mean[Metric7] & 0.0235 & 0.0593 & 0.0295 & 0.0388 & 0.0731 & 0.0124 & 0.0217 & 0.0581 & 0.0164 & 0.0251 \\
\hline SD[Metric7] & 0.0028 & 0.0079 & 0.0045 & 0.0054 & 0.0093 & 0.0011 & 0.0029 & 0.0084 & 0.0018 & 0.0031 \\
\hline Mean[Metric7-Bi] & 0.0073 & 0.0182 & 0.0033 & 0.0093 & 0.0245 & 0.0020 & 0.0046 & 0.0159 & 0.0025 & 0.0060 \\
\hline SD[Metric7-Bi] & 0.0034 & 0.0099 & 0.0013 & 0.0039 & 0.0109 & 0.0013 & 0.0033 & 0.0102 & 0.0013 & 0.0036 \\
\hline Mean[ Metric7-Bi/Bi] & 0.4803 & 0.4712 & 0.1320 & 0.3290 & 0.5318 & 0.2009 & 0.2865 & 0.4023 & 0.1881 & 0.3338 \\
\hline SD[ Metric7-Bi/Bi] & 0.2534 & 0.2846 & 0.0569 & 0.1605 & 0.2704 & 0.1300 & 0.2131 & 0.2818 & 0.1040 & 0.2129 \\
\hline SD[Metric7]/SDBi & 1.2387 & 1.2934 & 1.0205 & 1.0610 & 1.2865 & 1.3762 & 1.5345 & 1.4406 & 1.1169 & 1.3693 \\
\hline SD[Metric7-Bi]/SDBi & 1.5073 & 1.6148 & 0.2870 & 0.7659 & 1.5036 & 1.5777 & 1.7871 & 1.7516 & 0.8508 & 1.5839 \\
\hline Mean[Metric8] & 0.0300 & 0.0808 & 0.0317 & 0.0458 & 0.0951 & 0.0151 & 0.0295 & 0.0821 & 0.0191 & 0.0331 \\
\hline SD[Metric8] & 0.0016 & 0.0025 & 0.0045 & 0.0047 & 0.0049 & 0.0005 & 0.0008 & 0.0020 & 0.0015 & 0.0016 \\
\hline Mean[Metric8-Bi] & 0.0139 & 0.0396 & 0.0055 & 0.0162 & 0.0465 & 0.0047 & 0.0125 & 0.0399 & 0.0052 & 0.0141 \\
\hline SD[Metric8-Bi] & 0.0024 & 0.0066 & 0.0010 & 0.0030 & 0.0076 & 0.0008 & 0.0020 & 0.0062 & 0.0010 & 0.0024 \\
\hline Mean[ Metric8-Bi/Bi] & 0.8929 & 1.0039 & 0.2175 & 0.5727 & 0.9953 & 0.4632 & 0.7537 & 0.9821 & 0.3827 & 0.7613 \\
\hline SD[ Metric8-Bi/Bi] & 0.2463 & 0.2910 & 0.0574 & 0.1645 & 0.2773 & 0.1080 & 0.1914 & 0.2773 & 0.0946 & 0.1976 \\
\hline SD[Metric8]/SDBi & 0.6893 & 0.4089 & 1.0064 & 0.9283 & 0.6737 & 0.6713 & 0.4190 & 0.3474 & 0.9560 & 0.6842 \\
\hline SD[Metric8-Bi]/SDBi & 1.0434 & 1.0736 & 0.2300 & 0.5843 & 1.0517 & 1.0334 & 1.0673 & 1.0650 & 0.6213 & 1.0387 \\
\hline Mean[Metric9] & 0.0223 & 0.0571 & 0.0290 & 0.0375 & 0.0703 & 0.0124 & 0.0216 & 0.0567 & 0.0164 & 0.0249 \\
\hline SD[Metric9] & 0.0030 & 0.0080 & 0.0045 & 0.0055 & 0.0094 & 0.0011 & 0.0030 & 0.0087 & 0.0018 & 0.0032 \\
\hline Mean[Metric9-Bi] & 0.0061 & 0.0159 & 0.0028 & 0.0080 & 0.0218 & 0.0020 & 0.0045 & 0.0145 & 0.0025 & 0.0059 \\
\hline SD[Metric9-Bi] & 0.0036 & 0.0099 & 0.0014 & 0.0041 & 0.0111 & 0.0013 & 0.0034 & 0.0105 & 0.0013 & 0.0037 \\
\hline Mean[ Metric9-Bi/Bi] & 0.4041 & 0.4155 & 0.1119 & 0.2851 & 0.4750 & 0.2009 & 0.2804 & 0.3689 & 0.1877 & 0.3261 \\
\hline SD[ Metric9-Bi/Bi] & 0.2569 & 0.2783 & 0.0599 & 0.1631 & 0.2694 & 0.1300 & 0.2181 & 0.2844 & 0.1039 & 0.2171 \\
\hline SD[Metric9]/SDBi & 1.3429 & 1.3059 & 1.0160 & 1.0752 & 1.2905 & 1.3762 & 1.5961 & 1.4912 & 1.1204 & 1.4204 \\
\hline SD[Metric9-Bi]/SDBi & 1.5995 & 1.6151 & 0.3091 & 0.8027 & 1.5321 & 1.5777 & 1.8410 & 1.7936 & 0.8512 & 1.6241 \\
\hline formMean & 0.0109 & 0.0364 & 0.0036 & 0.0111 & 0.0370 & 0.0037 & 0.0109 & 0.0365 & 0.0036 & 0.0109 \\
\hline formStdDev & 0.0021 & 0.0066 & 0.0007 & 0.0022 & 0.0070 & 0.0007 & 0.0020 & 0.0069 & 0.0007 & 0.0021 \\
\hline Points per sphere & 25 & 25 & 25 & 25 & 25 & 25 & 25 & 25 & 25 & 25 \\
\hline Scenario number & 32 & 33 & 34 & 35 & 36 & 37 & 38 & 39 & 40 & 41 \\
\hline
\end{tabular}




\begin{tabular}{|c|c|c|c|c|c|c|c|c|c|c|}
\hline Scenario number & 42 & 43 & 44 & 45 & 46 & 47 & 48 & 49 & 50 & 51 \\
\hline IongRangeErrorDist & $\mathrm{N}[0.003,0.003]$ & $\mathrm{N}[0.003,0.010]$ & $\mathrm{N}[0.003,0.010]$ & $\mathrm{N}[0.003,0.010]$ & $\mathrm{N}[0.003,0.001]$ & $\mathrm{N}[0.003,0.001]$ & $\mathrm{N}[0.003,0.001]$ & $\mathrm{N}[0.003,0.003]$ & $\mathrm{N}[0.003,0.003]$ & $\mathrm{N}[0.003,0.003]$ \\
\hline probeRndErrorDist & $\mathrm{N}[0.0015,0.010]$ & $\mathrm{N}[0.0015,0.001]$ & $\mathrm{N}[0.0015,0.003]$ & $\mathrm{N}[0.0015,0.010]$ & $\mathrm{N}[0.005,0.001]$ & $\mathrm{N}[0.005,0.003]$ & $\mathrm{N}[0.005,0.010]$ & $\mathrm{N}[0.005,0.001]$ & $\mathrm{N}[0.005,0.003]$ & $\mathrm{N}[0.005,0.010]$ \\
\hline Mean[Bi] & 0.0427 & 0.0286 & 0.0319 & 0.0501 & 0.0173 & 0.0240 & 0.0487 & 0.0208 & 0.0261 & 0.0497 \\
\hline stdDevBi & 0.0060 & 0.0048 & 0.0052 & 0.0073 & 0.0008 & 0.0018 & 0.0058 & 0.0015 & 0.0023 & 0.0063 \\
\hline Mean[Metric1] & 0.0441 & 0.0286 & 0.0317 & 0.0512 & 0.0168 & 0.0235 & 0.0501 & 0.0204 & 0.0257 & 0.0508 \\
\hline SD[Metric1] & 0.0089 & 0.0046 & 0.0050 & 0.0093 & 0.0009 & 0.0027 & 0.0092 & 0.0015 & 0.0027 & 0.0092 \\
\hline Mean[Metric1-Bi] & 0.0013 & 0.0000 & -0.0002 & 0.0011 & -0.0006 & -0.0005 & 0.0014 & -0.0004 & -0.0004 & 0.0011 \\
\hline SD[Metric1-Bi] & 0.0106 & 0.0021 & 0.0039 & 0.0114 & 0.0011 & 0.0032 & 0.0109 & 0.0012 & 0.0034 & 0.0112 \\
\hline Mean[ Metric1-Bi/Bi] & 0.0504 & 0.0056 & 0.0013 & 0.0415 & -0.0313 & -0.0172 & 0.0431 & -0.0176 & -0.0074 & 0.0377 \\
\hline SD[ Metric1-Bi/Bi] & 0.2559 & 0.0797 & 0.1274 & 0.2361 & 0.0610 & 0.1331 & 0.2297 & 0.0590 & 0.1296 & 0.2298 \\
\hline SD[Metric1]/SDBi & 1.4871 & 0.9573 & 0.9472 & 1.2844 & 1.1203 & 1.4345 & 1.5815 & 1.0115 & 1.1923 & 1.4741 \\
\hline SD[Metric1-Bi]/SDBi & 1.7684 & 0.4237 & 0.7486 & 1.5760 & 1.3607 & 1.7318 & 1.8762 & 0.8369 & 1.5036 & 1.7934 \\
\hline Mean[Metric2] & 0.0695 & 0.0302 & 0.0374 & 0.0760 & 0.0189 & 0.0308 & 0.0762 & 0.0223 & 0.0327 & 0.0762 \\
\hline SD[Metric2] & 0.0008 & 0.0046 & 0.0042 & 0.0027 & 0.0004 & 0.0004 & 0.0007 & 0.0012 & 0.0009 & 0.0009 \\
\hline Mean[Metric2-Bi] & 0.0268 & 0.0016 & 0.0055 & 0.0259 & 0.0016 & 0.0068 & 0.0275 & 0.0015 & 0.0067 & 0.0265 \\
\hline SD[Metric2-Bi] & 0.0060 & 0.0019 & 0.0033 & 0.0071 & 0.0008 & 0.0019 & 0.0058 & 0.0009 & 0.0023 & 0.0063 \\
\hline Mean[ Metric2-Bi/Bi] & 0.6577 & 0.0617 & 0.1869 & 0.5471 & 0.0923 & 0.2916 & 0.5876 & 0.0726 & 0.2638 & 0.5574 \\
\hline SD[ Metric2-Bi/Bi] & 0.2297 & 0.0799 & 0.1273 & 0.2125 & 0.0469 & 0.0976 & 0.1876 & 0.0476 & 0.1054 & 0.1914 \\
\hline SD[Metric2]/SDBi & 0.1364 & 0.9411 & 0.8046 & 0.3739 & 0.5089 & 0.1961 & 0.1195 & 0.8405 & 0.4098 & 0.1391 \\
\hline SD[Metric2-Bi]/SDBi & 1.0065 & 0.3980 & 0.6299 & 0.9747 & 0.9664 & 1.0038 & 1.0028 & 0.6350 & 1.0000 & 1.0049 \\
\hline Mean[Metric3] & 0.0432 & 0.0279 & 0.0310 & 0.0509 & 0.0161 & 0.0209 & 0.0455 & 0.0201 & 0.0239 & 0.0463 \\
\hline SD[Metric3] & 0.0066 & 0.0048 & 0.0050 & 0.0073 & 0.0008 & 0.0021 & 0.0070 & 0.0015 & 0.0024 & 0.0070 \\
\hline Mean[Metric3-Bi] & 0.0005 & -0.0006 & -0.0009 & 0.0008 & -0.0012 & -0.0032 & -0.0032 & -0.0007 & -0.0022 & -0.0034 \\
\hline SD[Metric3-Bi] & 0.0090 & 0.0013 & 0.0035 & 0.0098 & 0.0010 & 0.0028 & 0.0091 & 0.0012 & 0.0032 & 0.0095 \\
\hline Mean[ Metric3-Bi/Bi] & 0.0317 & -0.0211 & -0.0227 & 0.0348 & -0.0697 & -0.1267 & -0.0529 & -0.0342 & -0.0783 & -0.0535 \\
\hline SD[ Metric3-Bi/Bi] & 0.2161 & 0.0467 & 0.1112 & 0.1993 & 0.0567 & 0.1088 & 0.1852 & 0.0575 & 0.1158 & 0.1860 \\
\hline SD[Metric3]/SDBi & 1.0988 & 0.9922 & 0.9569 & 1.0004 & 1.0547 & 1.1322 & 1.1958 & 1.0339 & 1.0759 & 1.1173 \\
\hline SD[Metric3-Bi]/SDBi & 1.5057 & 0.2709 & 0.6746 & 1.3444 & 1.2933 & 1.4884 & 1.5653 & 0.8216 & 1.3936 & 1.5100 \\
\hline Mean[Metric4] & 0.0444 & 0.0281 & 0.0315 & 0.0515 & 0.0164 & 0.0217 & 0.0480 & 0.0203 & 0.0246 & 0.0485 \\
\hline SD[Metric4] & 0.0060 & 0.0048 & 0.0048 & 0.0067 & 0.0006 & 0.0013 & 0.0056 & 0.0014 & 0.0018 & 0.0055 \\
\hline Mean[Metric4-Bi] & 0.0017 & -0.0005 & -0.0005 & 0.0014 & -0.0010 & -0.0023 & -0.0006 & -0.0006 & -0.0015 & -0.0012 \\
\hline SD[Metric4-Bi] & 0.0086 & 0.0012 & 0.0033 & 0.0093 & 0.0008 & 0.0023 & 0.0081 & 0.0010 & 0.0027 & 0.0084 \\
\hline Mean[ Metric4-Bi/Bi] & 0.0600 & -0.0150 & -0.0070 & 0.0478 & -0.0544 & -0.0898 & 0.0008 & -0.0251 & -0.0508 & -0.0089 \\
\hline SD[ Metric4-Bi/Bi] & 0.2075 & 0.0432 & 0.1031 & 0.1918 & 0.0457 & 0.0879 & 0.1686 & 0.0476 & 0.0975 & 0.1672 \\
\hline SD[Metric4]/SDBi & 0.9926 & 0.9879 & 0.9106 & 0.9212 & 0.7122 & 0.7246 & 0.9603 & 0.9369 & 0.8026 & 0.8797 \\
\hline SD[Metric4-Bi]/SDBi & 1.4296 & 0.2486 & 0.6236 & 1.2783 & 1.0548 & 1.2229 & 1.3927 & 0.6878 & 1.1806 & 1.3344 \\
\hline Mean[Metric5] & 0.0560 & 0.0283 & 0.0331 & 0.0625 & 0.0167 & 0.0241 & 0.0591 & 0.0206 & 0.0267 & 0.0590 \\
\hline SD[Metric5] & 0.0014 & 0.0048 & 0.0045 & 0.0034 & 0.0005 & 0.0007 & 0.0016 & 0.0014 & 0.0014 & 0.0018 \\
\hline Mean[Metric5-Bi] & 0.0133 & -0.0002 & 0.0012 & 0.0124 & -0.0006 & 0.0000 & 0.0104 & -0.0003 & 0.0006 & 0.0093 \\
\hline SD[Metric5-Bi] & 0.0061 & 0.0012 & 0.0031 & 0.0072 & 0.0008 & 0.0020 & 0.0060 & 0.0010 & 0.0024 & 0.0065 \\
\hline Mean[ Metric5-Bi/Bi] & 0.3365 & -0.0071 & 0.0472 & 0.2717 & -0.0335 & 0.0072 & 0.2305 & -0.0120 & 0.0308 & 0.2058 \\
\hline SD[ Metric5-Bi/Bi] & 0.1849 & 0.0431 & 0.1030 & 0.1777 & 0.0456 & 0.0807 & 0.1484 & 0.0471 & 0.0924 & 0.1527 \\
\hline SD[Metric5]/SDBi & 0.2396 & 0.9810 & 0.8593 & 0.4687 & 0.6755 & 0.3816 & 0.2691 & 0.9207 & 0.6024 & 0.2938 \\
\hline SD[Metric5-Bi]/SDBi & 1.0106 & 0.2471 & 0.5972 & 0.9950 & 1.0397 & 1.0590 & 1.0296 & 0.6764 & 1.0639 & 1.0389 \\
\hline Mean[Metric6] & 0.0606 & 0.0294 & 0.0359 & 0.0690 & 0.0177 & 0.0263 & 0.0639 & 0.0217 & 0.0292 & 0.0645 \\
\hline SD[Metric6] & 0.0014 & 0.0048 & 0.0046 & 0.0037 & 0.0005 & 0.0006 & 0.0014 & 0.0014 & 0.0013 & 0.0016 \\
\hline Mean[Metric6-Bi] & 0.0178 & 0.0008 & 0.0040 & 0.0189 & 0.0004 & 0.0022 & 0.0152 & 0.0008 & 0.0032 & 0.0148 \\
\hline SD[Metric6-Bi] & 0.0061 & 0.0012 & 0.0031 & 0.0072 & 0.0008 & 0.0019 & 0.0059 & 0.0010 & 0.0024 & 0.0064 \\
\hline Mean[ Metric6-Bi/Bi] & 0.4450 & 0.0302 & 0.1371 & 0.4025 & 0.0229 & 0.0993 & 0.3313 & 0.0419 & 0.1285 & 0.3180 \\
\hline SD[ Metric6-Bi/Bi] & 0.2019 & 0.0446 & 0.1103 & 0.1939 & 0.0459 & 0.0853 & 0.1588 & 0.0470 & 0.0981 & 0.1643 \\
\hline SD[Metric6]/SDBi & 0.2291 & 0.9910 & 0.8887 & 0.5051 & 0.6468 & 0.3501 & 0.2376 & 0.9163 & 0.5856 & 0.2624 \\
\hline SD[Metric6-Bi]/SDBi & 1.0205 & 0.2495 & 0.5935 & 0.9950 & 1.0040 & 1.0332 & 1.0196 & 0.6481 & 1.0492 & 1.0255 \\
\hline Mean[Metric7] & 0.0602 & 0.0320 & 0.0405 & 0.0729 & 0.0193 & 0.0286 & 0.0629 & 0.0234 & 0.0320 & 0.0646 \\
\hline SD[Metric7] & 0.0085 & 0.0047 & 0.0056 & 0.0098 & 0.0011 & 0.0030 & 0.0095 & 0.0017 & 0.0033 & 0.0096 \\
\hline Mean[Metric7-Bi] & 0.0174 & 0.0034 & 0.0086 & 0.0229 & 0.0020 & 0.0046 & 0.0142 & 0.0025 & 0.0060 & 0.0148 \\
\hline SD[Metric7-Bi] & 0.0105 & 0.0021 & 0.0044 & 0.0117 & 0.0012 & 0.0035 & 0.0111 & 0.0014 & 0.0038 & 0.0115 \\
\hline Mean[ Metric7-Bi/Bi] & 0.4359 & 0.1271 & 0.2831 & 0.4835 & 0.1150 & 0.1974 & 0.3106 & 0.1244 & 0.2377 & 0.3191 \\
\hline SD[ Metric7-Bi/Bi] & 0.2886 & 0.0914 & 0.1645 & 0.2778 & 0.0739 & 0.1526 & 0.2522 & 0.0716 & 0.1572 & 0.2563 \\
\hline SD[Metric7]/SDBi & 1.4248 & 0.9737 & 1.0620 & 1.3527 & 1.3951 & 1.6075 & 1.6299 & 1.1487 & 1.4521 & 1.5345 \\
\hline SD[Metric7-Bi]/SDBi & 1.7527 & 0.4428 & 0.8363 & 1.6057 & 1.5663 & 1.8684 & 1.9102 & 0.9591 & 1.6899 & 1.8387 \\
\hline Mean[Metric8] & 0.0841 & 0.0347 & 0.0484 & 0.0981 & 0.0220 & 0.0366 & 0.0897 & 0.0261 & 0.0401 & 0.0909 \\
\hline SD[Metric8] & 0.0024 & 0.0046 & 0.0048 & 0.0051 & 0.0005 & 0.0008 & 0.0022 & 0.0014 & 0.0015 & 0.0025 \\
\hline Mean[Metric8-Bi] & 0.0414 & 0.0061 & 0.0165 & 0.0480 & 0.0047 & 0.0126 & 0.0410 & 0.0052 & 0.0141 & 0.0412 \\
\hline SD[Metric8-Bi] & 0.0064 & 0.0019 & 0.0034 & 0.0077 & 0.0008 & 0.0020 & 0.0061 & 0.0010 & 0.0025 & 0.0067 \\
\hline Mean[ Metric8-Bi/Bi] & 1.0065 & 0.2251 & 0.5390 & 0.9941 & 0.2726 & 0.5320 & 0.8677 & 0.2531 & 0.5508 & 0.8561 \\
\hline SD[ Metric8-Bi/Bi] & 0.2826 & 0.0953 & 0.1685 & 0.2760 & 0.0555 & 0.1179 & 0.2235 & 0.0557 & 0.1319 & 0.2321 \\
\hline SD[Metric8]/SDBi & 0.3977 & 0.9596 & 0.9205 & 0.6995 & 0.6745 & 0.4267 & 0.3790 & 0.9402 & 0.6833 & 0.3989 \\
\hline SD[Metric8-Bi]/SDBi & 1.0693 & 0.3999 & 0.6471 & 1.0658 & 1.0135 & 1.0560 & 1.0547 & 0.6535 & 1.0882 & 1.0641 \\
\hline Mean[Metric9] & 0.0588 & 0.0314 & 0.0398 & 0.0713 & 0.0193 & 0.0286 & 0.0626 & 0.0234 & 0.0320 & 0.0643 \\
\hline SD[Metric9] & 0.0088 & 0.0049 & 0.0057 & 0.0100 & 0.0011 & 0.0030 & 0.0097 & 0.0017 & 0.0033 & 0.0099 \\
\hline Mean[Metric9-Bi] & 0.0161 & 0.0028 & 0.0079 & 0.0212 & 0.0020 & 0.0046 & 0.0139 & 0.0025 & 0.0060 & 0.0146 \\
\hline SD[Metric9-Bi] & 0.0107 & 0.0015 & 0.0041 & 0.0118 & 0.0012 & 0.0035 & 0.0114 & 0.0014 & 0.0038 & 0.0117 \\
\hline Mean[ Metric9-Bi/Bi] & 0.4043 & 0.1013 & 0.2610 & 0.4490 & 0.1150 & 0.1974 & 0.3047 & 0.1243 & 0.2375 & 0.3136 \\
\hline SD[ Metric9-Bi/Bi] & 0.2884 & 0.0585 & 0.1508 & 0.2765 & 0.0739 & 0.1526 & 0.2561 & 0.0716 & 0.1573 & 0.2598 \\
\hline SD[Metric9]/SDBi & 1.4644 & 1.0095 & 1.0824 & 1.3795 & 1.3951 & 1.6075 & 1.6719 & 1.1482 & 1.4540 & 1.5743 \\
\hline SD[Metric9-Bi]/SDBi & 1.7825 & 0.3071 & 0.7899 & 1.6204 & 1.5663 & 1.8684 & 1.9504 & 0.9584 & 1.6914 & 1.8729 \\
\hline formMean & 0.0366 & 0.0037 & 0.0110 & 0.0365 & 0.0036 & 0.0110 & 0.0368 & 0.0036 & 0.0109 & 0.0364 \\
\hline formStdDev & 0.0068 & 0.0007 & 0.0021 & 0.0073 & 0.0007 & 0.0021 & 0.0069 & 0.0007 & 0.0021 & 0.0067 \\
\hline Points per sphere & 25 & 25 & 25 & 25 & 25 & 25 & 25 & 25 & 25 & 25 \\
\hline Scenario number & 42 & 43 & 44 & 45 & 46 & 47 & 48 & 49 & 50 & 51 \\
\hline
\end{tabular}


16

\begin{tabular}{|c|c|c|c|c|c|c|c|c|c|c|}
\hline Scenario number & 52 & 53 & 54 & 55 & 56 & 57 & 58 & 59 & 60 & 61 \\
\hline IongRangeErrorDist & $\mathrm{N}[0.003,0.010]$ & $\mathrm{N}[0.003,0.010]$ & $\mathrm{N}[0.003,0.010]$ & $\mathrm{N}[0.010,0.001]$ & $\mathrm{N}[0.010,0.001]$ & $\mathrm{N}[0.010,0.001]$ & $\mathrm{N}[0.010,0.003]$ & $\mathrm{N}[0.010,0.003]$ & $\mathrm{N}[0.010,0.003]$ & $\mathrm{N}[0.010,0.010]$ \\
\hline probeRndErrorDist & $\mathrm{N}[0.005,0.001]$ & $\mathrm{N}[0.005,0.003]$ & $\mathrm{N}[0.005,0.010]$ & $\mathrm{N}[0 ., 0.001]$ & $\mathrm{N}[0 ., 0.003]$ & $\mathrm{N}[0 ., 0.010]$ & $\mathrm{N}[0 ., 0.001]$ & $\mathrm{N}[0 ., 0.003]$ & $\mathrm{N}[0 ., 0.010]$ & $\mathrm{N}[0 ., 0.001]$ \\
\hline Mean[Bi] & 0.0354 & 0.0386 & 0.0565 & 0.0143 & 0.0210 & \begin{tabular}{|l|}
0.0461 \\
\end{tabular} & \begin{tabular}{|l|l|}
0.0179 \\
\end{tabular} & 0.0230 & 0.0467 & 0.0327 \\
\hline stdDevBi & 0.0048 & 0.0052 & 0.0080 & 0.0008 & 0.0019 & 0.0062 & 0.0015 & 0.0023 & 0.0064 & 0.0047 \\
\hline Mean[Metric1] & 0.0355 & 0.0385 & 0.0585 & 0.0132 & 0.0183 & 0.0431 & 0.0172 & 0.0210 & 0.0447 & 0.0320 \\
\hline SD[Metric1] & 0.0045 & 0.0050 & 0.0093 & 0.0009 & 0.0024 & 0.0092 & 0.0016 & 0.0027 & 0.0088 & 0.0047 \\
\hline Mean[Metric1-Bi] & 0.0001 & -0.0001 & 0.0020 & -0.0011 & -0.0027 & -0.0030 & -0.0007 & -0.0020 & -0.0020 & -0.0007 \\
\hline SD[Metric1-Bi] & 0.0025 & 0.0040 & 0.0120 & 0.0011 & 0.0031 & 0.0109 & 0.0012 & 0.0033 & 0.0108 & 0.0013 \\
\hline Mean[ Metric1-Bi/Bi] & 0.0075 & 0.0048 & 0.0551 & -0.0782 & -0.1224 & -0.0487 & -0.0377 & -0.0804 & -0.0255 & -0.0213 \\
\hline SD[ Metric1-Bi/Bi] & 0.0832 & 0.1095 & 0.2168 & 0.0725 & 0.1384 & 0.2344 & 0.0644 & 0.1399 & 0.2295 & 0.0402 \\
\hline SD[Metric1]/SDBi & 0.9497 & 0.9472 & 1.1655 & 1.1405 & 1.2317 & 1.4700 & 1.0670 & 1.1673 & 1.3869 & 1.0079 \\
\hline SD[Metric1-Bi]/SDBi & 0.5318 & 0.7699 & 1.4919 & 1.4136 & 1.5762 & 1.7557 & 0.7719 & 1.4400 & 1.7000 & 0.2751 \\
\hline Mean[Metric2] & 0.0370 & 0.0442 & 0.0828 & 0.0150 & 0.0248 & 0.0689 & 0.0189 & 0.0278 & 0.0699 & 0.0335 \\
\hline SD[Metric2] & 0.0044 & 0.0042 & 0.0027 & 0.0005 & 0.0005 & 0.0010 & 0.0014 & 0.0012 & 0.0012 & 0.0046 \\
\hline Mean[Metric2-Bi] & 0.0016 & 0.0056 & 0.0263 & 0.0007 & 0.0038 & 0.0228 & 0.0010 & 0.0048 & 0.0232 & 0.0009 \\
\hline SD[Metric2-Bi] & 0.0024 & 0.0035 & 0.0079 & 0.0008 & 0.0020 & 0.0063 & 0.0010 & 0.0023 & 0.0064 & 0.0011 \\
\hline Mean[ Metric2-Bi/Bi] & 0.0505 & 0.1566 & 0.4926 & 0.0499 & 0.1905 & 0.5219 & 0.0580 & 0.2176 & 0.5234 & 0.0277 \\
\hline SD[ Metric2-Bi/Bi] & 0.0826 & 0.1081 & 0.2009 & 0.0551 & 0.1098 & 0.1996 & 0.0563 & 0.1142 & 0.1990 & 0.0352 \\
\hline SD[Metric2]/SDBi & 0.9313 & 0.8005 & 0.3320 & 0.6456 & 0.2746 & 0.1566 & 0.9509 & 0.5127 & 0.1834 & 0.9841 \\
\hline SD[Metric2-Bi]/SDBi & 0.5094 & 0.6613 & 0.9872 & 1.0116 & 1.0417 & 1.0090 & 0.6403 & 0.9908 & 1.0150 & 0.2345 \\
\hline Mean[Metric3] & 0.0347 & 0.0372 & 0.0551 & 0.0132 & 0.0183 & 0.0437 & 0.0172 & 0.0211 & 0.0451 & 0.0320 \\
\hline SD[Metric3] & 0.0048 & 0.0052 & 0.0079 & 0.0008 & 0.0021 & 0.0070 & 0.0016 & 0.0025 & 0.0070 & 0.0047 \\
\hline Mean[Metric3-Bi] & -0.0007 & -0.0014 & -0.0013 & -0.0011 & -0.0027 & -0.0024 & -0.0007 & -0.0020 & -0.0016 & -0.0007 \\
\hline SD[Metric3-Bi] & 0.0013 & 0.0034 & 0.0107 & 0.0011 & 0.0028 & 0.0092 & 0.0012 & 0.0031 & 0.0093 & 0.0013 \\
\hline Mean[ Metric3-Bi/Bi] & -0.0195 & -0.0326 & -0.0068 & -0.0779 & -0.1209 & -0.0349 & -0.0376 & -0.0783 & -0.0180 & -0.0213 \\
\hline SD[ Metric3-Bi/Bi] & 0.0364 & 0.0888 & 0.1881 & 0.0712 & 0.1263 & 0.1967 & 0.0639 & 0.1315 & 0.1975 & 0.0399 \\
\hline SD[Metric3]/SDBi & 1.0030 & 0.9985 & 0.9880 & 1.1137 & 1.0735 & 1.1199 & 1.0626 & 1.0667 & 1.1003 & 1.0083 \\
\hline SD[Metric3-Bi]/SDBi & 0.2668 & 0.6557 & 1.3339 & 1.3912 & 1.4524 & 1.4810 & 0.7664 & 1.3614 & 1.4717 & 0.2738 \\
\hline Mean[Metric4] & 0.0349 & 0.0378 & 0.0568 & 0.0135 & 0.0192 & 0.0460 & 0.0174 & 0.0218 & 0.0470 & 0.0321 \\
\hline SD[Metric4] & 0.0047 & 0.0049 & 0.0065 & 0.0006 & 0.0014 & 0.0059 & 0.0015 & 0.0017 & 0.0060 & 0.0046 \\
\hline Mean[Metric4-Bi] & -0.0005 & -0.0008 & 0.0004 & -0.0008 & -0.0018 & -0.0001 & -0.0005 & -0.0012 & 0.0003 & -0.0005 \\
\hline SD[Metric4-Bi] & 0.0011 & 0.0031 & 0.0094 & 0.0009 & 0.0023 & 0.0085 & 0.0010 & 0.0026 & 0.0086 & 0.0012 \\
\hline Mean[ Metric4-Bi/Bi] & -0.0132 & -0.0157 & 0.0232 & -0.0562 & -0.0791 & 0.0158 & -0.0236 & -0.0438 & 0.0240 & -0.0155 \\
\hline SD[ Metric4-Bi/Bi] & 0.0325 & 0.0798 & 0.1687 & 0.0565 & 0.1033 & 0.1855 & 0.0570 & 0.1087 & 0.1851 & 0.0354 \\
\hline SD[Metric4]/SDBi & 0.9904 & 0.9283 & 0.8113 & 0.7441 & 0.7238 & 0.9524 & 0.9778 & 0.7501 & 0.9486 & 0.9888 \\
\hline SD[Metric4-Bi]/SDBi & 0.2385 & 0.5911 & 1.1711 & 1.1186 & 1.1935 & 1.3705 & 0.6811 & 1.1351 & 1.3590 & 0.2447 \\
\hline Mean[Metric5] & 0.0351 & 0.0392 & 0.0667 & 0.0139 & 0.0217 & 0.0577 & 0.0178 & 0.0241 & 0.0587 & 0.0323 \\
\hline SD[Metric5] & 0.0047 & 0.0047 & 0.0038 & 0.0005 & 0.0007 & 0.0015 & 0.0014 & 0.0012 & 0.0016 & 0.0046 \\
\hline Mean[Metric5-Bi] & -0.0003 & 0.0006 & 0.0102 & -0.0004 & 0.0007 & 0.0116 & -0.0001 & 0.0011 & 0.0121 & -0.0003 \\
\hline SD[Metric5-Bi] & 0.0011 & 0.0030 & 0.0080 & 0.0008 & 0.0021 & 0.0064 & 0.0010 & 0.0023 & 0.0066 & 0.0011 \\
\hline Mean[ Metric5-Bi/Bi] & -0.0083 & 0.0203 & 0.2015 & -0.0264 & 0.0415 & 0.2729 & -0.0049 & 0.0568 & 0.2807 & -0.0095 \\
\hline SD[ Metric5-Bi/Bi] & 0.0324 & 0.0803 & 0.1630 & 0.0543 & 0.0983 & 0.1684 & 0.0564 & 0.1024 & 0.1700 & 0.0352 \\
\hline SD[Metric5]/SDBi & 0.9853 & 0.8919 & 0.4704 & 0.6753 & 0.3580 & 0.2377 & 0.9574 & 0.5296 & 0.2496 & 0.9831 \\
\hline SD[Metric5-Bi]/SDBi & 0.2378 & 0.5789 & 0.9937 & 1.0578 & 1.0536 & 1.0227 & 0.6674 & 1.0093 & 1.0351 & 0.2433 \\
\hline Mean[Metric6] & 0.0361 & 0.0422 & 0.0736 & 0.0149 & 0.0238 & 0.0623 & 0.0188 & 0.0269 & 0.0636 & 0.0335 \\
\hline SD[Metric6] & 0.0047 & 0.0047 & 0.0038 & 0.0005 & 0.0006 & 0.0014 & 0.0014 & 0.0013 & 0.0016 & 0.0046 \\
\hline Mean[Metric6-Bi] & 0.0007 & 0.0036 & 0.0172 & 0.0006 & 0.0028 & 0.0162 & 0.0009 & 0.0039 & 0.0169 & 0.0008 \\
\hline SD[Metric6-Bi] & 0.0011 & 0.0028 & 0.0080 & 0.0008 & 0.0020 & 0.0063 & 0.0010 & 0.0023 & 0.0065 & 0.0011 \\
\hline Mean[ Metric6-Bi/Bi] & 0.0212 & 0.1002 & 0.3270 & 0.0437 & 0.1410 & 0.3757 & 0.0550 & 0.1793 & 0.3861 & 0.0269 \\
\hline SD[ Metric6-Bi/Bi] & 0.0315 & 0.0817 & 0.1798 & 0.0550 & 0.1063 & 0.1816 & 0.0563 & 0.1117 & 0.1826 & 0.0352 \\
\hline SD[Metric6]/SDBi & 0.9911 & 0.8965 & 0.4720 & 0.6536 & 0.3056 & 0.2169 & 0.9563 & 0.5533 & 0.2470 & 0.9859 \\
\hline SD[Metric6-Bi]/SDBi & 0.2267 & 0.5450 & 1.0025 & 1.0143 & 1.0508 & 1.0191 & 0.6418 & 1.0012 & 1.0276 & 0.2353 \\
\hline Mean[Metric7] & 0.0389 & 0.0472 & 0.0781 & 0.0169 & 0.0271 & 0.0648 & 0.0210 & 0.0306 & 0.0666 & 0.0360 \\
\hline SD[Metric7] & 0.0047 & 0.0056 & 0.0108 & 0.0010 & 0.0024 & 0.0083 & 0.0017 & 0.0028 & 0.0086 & 0.0047 \\
\hline Mean[Metric7-Bi] & 0.0035 & 0.0086 & 0.0216 & 0.0025 & 0.0060 & 0.0187 & 0.0031 & 0.0076 & 0.0199 & 0.0033 \\
\hline SD[Metric7-Bi] & 0.0027 & 0.0044 & 0.0129 & 0.0012 & 0.0030 & 0.0103 & 0.0013 & 0.0034 & 0.0105 & 0.0014 \\
\hline Mean[ Metric7-Bi/Bi] & 0.1036 & 0.2323 & 0.4077 & 0.1801 & 0.2972 & 0.4293 & 0.1764 & 0.3409 & 0.4511 & 0.1038 \\
\hline SD[ Metric7-Bi/Bi] & 0.0922 & 0.1344 & 0.2644 & 0.0869 & 0.1609 & 0.2596 & 0.0755 & 0.1660 & 0.2618 & 0.0474 \\
\hline SD[Metric7]/SDBi & 0.9776 & 1.0642 & 1.3495 & 1.2773 & 1.2425 & 1.3325 & 1.1036 & 1.2137 & 1.3470 & 1.0076 \\
\hline SD[Metric7-Bi]/SDBi & 0.5586 & 0.8466 & 1.6136 & 1.5362 & 1.5609 & 1.6500 & 0.8301 & 1.4516 & 1.6478 & 0.2925 \\
\hline Mean[Metric8] & 0.0415 & 0.0552 & 0.1048 & 0.0191 & 0.0335 & 0.0869 & 0.0232 & 0.0374 & 0.0889 & 0.0382 \\
\hline SD[Metric8] & 0.0045 & 0.0048 & 0.0049 & 0.0005 & 0.0008 & 0.0023 & 0.0015 & 0.0015 & 0.0026 & 0.0047 \\
\hline Mean[Metric8-Bi] & 0.0061 & 0.0166 & 0.0483 & 0.0048 & 0.0124 & 0.0409 & 0.0053 & 0.0144 & 0.0422 & 0.0055 \\
\hline SD[Metric8-Bi] & 0.0024 & 0.0035 & 0.0085 & 0.0008 & 0.0021 & 0.0066 & 0.0010 & 0.0024 & 0.0068 & 0.0011 \\
\hline Mean[ Metric8-Bi/Bi] & 0.1795 & 0.4441 & 0.8889 & 0.3355 & 0.6049 & 0.9193 & 0.2998 & 0.6378 & 0.9373 & 0.1735 \\
\hline SD[ Metric8-Bi/Bi] & 0.0920 & 0.1357 & 0.2573 & 0.0683 & 0.1485 & 0.2548 & 0.0680 & 0.1533 & 0.2568 & 0.0452 \\
\hline SD[Metric8]/SDBi & 0.9497 & 0.9147 & 0.6140 & 0.6937 & 0.3926 & 0.3660 & 0.9915 & 0.6674 & 0.4100 & 0.9967 \\
\hline SD[Metric8-Bi]/SDBi & 0.5122 & 0.6785 & 1.0647 & 1.0288 & 1.0806 & 1.0585 & 0.6522 & 1.0385 & 1.0764 & 0.2355 \\
\hline Mean[Metric9] & 0.0381 & 0.0464 & 0.0773 & 0.0163 & 0.0255 & 0.0597 & 0.0204 & 0.0288 & 0.0620 & 0.0354 \\
\hline SD[Metric9] & 0.0049 & 0.0058 & 0.0110 & 0.0011 & 0.0029 & 0.0096 & 0.0018 & 0.0033 & 0.0096 & 0.0048 \\
\hline Mean[Metric9-Bi] & 0.0027 & 0.0079 & 0.0208 & 0.0020 & 0.0045 & 0.0136 & 0.0026 & 0.0058 & 0.0153 & 0.0028 \\
\hline SD[Metric9-Bi] & 0.0015 & 0.0040 & 0.0130 & 0.0013 & 0.0035 & 0.0112 & 0.0014 & 0.0038 & 0.0114 & 0.0015 \\
\hline Mean[ Metric9-Bi/Bi] & 0.0788 & 0.2106 & 0.3920 & 0.1406 & 0.2235 & 0.3180 & 0.1454 & 0.2618 & 0.3505 & 0.0866 \\
\hline SD[ Metric9-Bi/Bi] & 0.0441 & 0.1150 & 0.2633 & 0.0950 & 0.1757 & 0.2686 & 0.0803 & 0.1804 & 0.2716 & 0.0483 \\
\hline SD[Metric9]/SDBi & 1.0228 & 1.1054 & 1.3740 & 1.4637 & 1.5019 & 1.5360 & 1.1669 & 1.4445 & 1.5093 & 1.0241 \\
\hline SD[Metric9-Bi]/SDBi & 0.3045 & 0.7579 & 1.6214 & 1.7150 & 1.7805 & 1.8056 & 0.9033 & 1.6555 & 1.7974 & 0.3100 \\
\hline formMean & 0.0036 & 0.0110 & 0.0370 & 0.0037 & 0.0110 & 0.0365 & 0.0037 & 0.0110 & 0.0368 & 0.0037 \\
\hline formStdDev & 0.0007 & 0.0020 & 0.0070 & 0.0007 & 0.0020 & 0.0070 & 0.0007 & 0.0021 & 0.0072 & 0.0007 \\
\hline Points per sphere & 25 & 25 & 25 & 25 & 25 & 25 & 25 & 25 & 25 & 25 \\
\hline Scenario number & 52 & 53 & 54 & 55 & 56 & 57 & 58 & 59 & 60 & 61 \\
\hline
\end{tabular}


17

\begin{tabular}{|c|c|c|c|c|c|c|c|c|c|c|}
\hline Scenario number & 62 & 63 & 64 & 65 & 66 & 67 & 68 & 69 & 70 & 71 \\
\hline IongRangeErrorDist & $\mathrm{N}[0.010,0.010]$ & $\mathrm{N}[0.010,0.010]$ & $\mathrm{N}[0.010,0.001]$ & $\mathrm{N}[0.010,0.001]$ & $\mathrm{N}[0.010,0.001]$ & $\mathrm{N}[0.010,0.003]$ & $\mathrm{N}[0.010,0.003]$ & $\mathrm{N}[0.010,0.003]$ & $\mathrm{N}[0.010,0.010]$ & $\mathrm{N}[0.010,0.010]$ \\
\hline probeRndErrorDist & $\mathrm{N}[0 ., 0.003]$ & $\mathrm{N}[0 ., 0.010]$ & $\mathrm{N}[0.0015,0.001]$ & $\mathrm{N}[0.0015,0.003]$ & $\mathrm{N}[0.0015,0.010]$ & $\mathrm{N}[0.0015,0.001]$ & $\mathrm{N}[0.0015,0.003]$ & $\mathrm{N}[0.0015,0.010]$ & $\mathrm{N}[0.0015,0.001]$ & $\mathrm{N}[0.0015,0.003]$ \\
\hline Mean[Bi] & 0.0359 & 0.0535 & 0.0174 & 0.0240 & 0.0491 & 0.0210 & 0.0261 & 0.0495 & 0.0354 & 0.0384 \\
\hline stdDevBi & 0.0051 & 0.0074 & 0.0008 & 0.0018 & 0.0063 & 0.0016 & 0.0023 & 0.0062 & 0.0049 & 0.0050 \\
\hline Mean[Metric1] & 0.0346 & 0.0524 & 0.0163 & 0.0213 & 0.0464 & 0.0203 & 0.0242 & 0.0477 & 0.0348 & 0.0372 \\
\hline SD[Metric1] & 0.0051 & 0.0090 & 0.0009 & 0.0024 & 0.0089 & 0.0016 & 0.0027 & 0.0089 & 0.0050 & 0.0051 \\
\hline Mean[Metric1-Bi] & -0.0013 & -0.0010 & -0.0011 & -0.0026 & -0.0027 & -0.0007 & -0.0019 & -0.0018 & -0.0006 & -0.0012 \\
\hline SD[Metric1-Bi] & 0.0036 & 0.0111 & 0.0011 & 0.0030 & 0.0110 & 0.0012 & 0.0034 & 0.0107 & 0.0013 & 0.0035 \\
\hline Mean[ Metric1-Bi/Bi] & -0.0312 & -0.0031 & -0.0612 & -0.1038 & -0.0385 & -0.0306 & -0.0664 & -0.0226 & -0.0171 & -0.0270 \\
\hline SD[ Metric1-Bi/Bi] & 0.1013 & 0.2079 & 0.0590 & 0.1218 & 0.2239 & 0.0561 & 0.1233 & 0.2171 & 0.0369 & 0.0897 \\
\hline SD[Metric1]/SDBi & 0.9935 & 1.2049 & 1.1470 & 1.3109 & 1.3992 & 1.0164 & 1.1683 & 1.4237 & 1.0091 & 1.0220 \\
\hline SD[Metric1-Bi]/SDBi & 0.7158 & 1.4985 & 1.3980 & 1.6567 & 1.7381 & 0.7619 & 1.4284 & 1.7094 & 0.2598 & 0.7053 \\
\hline Mean[Metric2] & 0.0401 & 0.0752 & 0.0180 & 0.0279 & 0.0714 & 0.0219 & 0.0306 & 0.0724 & 0.0363 & 0.0427 \\
\hline SD[Metric2] & 0.0044 & 0.0032 & 0.0005 & 0.0006 & 0.0009 & 0.0014 & 0.0012 & 0.0011 & 0.0049 & 0.0044 \\
\hline Mean[Metric2-Bi] & 0.0041 & 0.0217 & 0.0007 & 0.0040 & 0.0223 & 0.0010 & 0.0045 & 0.0229 & 0.0009 & 0.0043 \\
\hline SD[Metric2-Bi] & 0.0030 & 0.0073 & 0.0008 & 0.0019 & 0.0064 & 0.0010 & 0.0023 & 0.0063 & 0.0011 & 0.0028 \\
\hline Mean[ Metric2-Bi/Bi] & 0.1236 & 0.4308 & 0.0402 & 0.1730 & 0.4783 & 0.0473 & 0.1822 & 0.4846 & 0.0262 & 0.1186 \\
\hline SD[ Metric2-Bi/Bi] & 0.0936 & 0.1883 & 0.0447 & 0.0891 & 0.1847 & 0.0477 & 0.1002 & 0.1815 & 0.0321 & 0.0797 \\
\hline SD[Metric2]/SDBi & 0.8681 & 0.4271 & 0.6516 & 0.3001 & 0.1434 & 0.9185 & 0.5109 & 0.1778 & 0.9903 & 0.8785 \\
\hline SD[Metric2-Bi]/SDBi & 0.5919 & 0.9781 & 1.0093 & 1.0306 & 1.0110 & 0.6243 & 0.9963 & 1.0153 & 0.2205 & 0.5605 \\
\hline Mean[Metric3] & 0.0346 & 0.0529 & 0.0162 & 0.0208 & 0.0455 & 0.0202 & 0.0238 & 0.0467 & 0.0347 & 0.0371 \\
\hline SD[Metric3] & 0.0050 & 0.0075 & 0.0009 & 0.0021 & 0.0069 & 0.0016 & 0.0025 & 0.0069 & 0.0050 & 0.0051 \\
\hline Mean[Metric3-Bi] & -0.0013 & -0.0006 & -0.0012 & -0.0031 & -0.0036 & -0.0007 & -0.0023 & -0.0029 & -0.0006 & -0.0013 \\
\hline SD[Metric3-Bi] & 0.0036 & 0.0099 & 0.0010 & 0.0028 & 0.0095 & 0.0012 & 0.0032 & 0.0091 & 0.0013 & 0.0034 \\
\hline Mean[ Metric3-Bi/Bi] & -0.0308 & 0.0057 & -0.0670 & -0.1252 & -0.0587 & -0.0334 & -0.0809 & -0.0438 & -0.0179 & -0.0315 \\
\hline SD[ Metric3-Bi/Bi] & 0.0992 & 0.1853 & 0.0579 & 0.1101 & 0.1881 & 0.0556 & 0.1155 & 0.1814 & 0.0365 & 0.0878 \\
\hline SD[Metric3]/SDBi & 0.9822 & 1.0040 & 1.1225 & 1.1435 & 1.0893 & 1.0171 & 1.0803 & 1.1023 & 1.0107 & 1.0201 \\
\hline SD[Metric3-Bi]/SDBi & 0.7036 & 1.3340 & 1.3758 & 1.5246 & 1.4944 & 0.7571 & 1.3541 & 1.4618 & 0.2573 & 0.6925 \\
\hline Mean[Metric4] & 0.0352 & 0.0543 & 0.0164 & 0.0218 & 0.0478 & 0.0204 & 0.0246 & 0.0487 & 0.0349 & 0.0376 \\
\hline SD[Metric4] & 0.0047 & 0.0064 & 0.0006 & 0.0014 & 0.0057 & 0.0015 & 0.0017 & 0.0055 & 0.0049 & 0.0046 \\
\hline Mean[Metric4-Bi] & -0.0008 & 0.0008 & -0.0009 & -0.0022 & -0.0013 & -0.0006 & -0.0015 & -0.0009 & -0.0005 & -0.0008 \\
\hline SD[Metric4-Bi] & 0.0033 & 0.0089 & 0.0008 & 0.0023 & 0.0084 & 0.0010 & 0.0026 & 0.0081 & 0.0011 & 0.0030 \\
\hline Mean[ Metric4-Bi/Bi] & -0.0161 & 0.0315 & -0.0525 & -0.0849 & -0.0118 & -0.0253 & -0.0517 & -0.0031 & -0.0130 & -0.0170 \\
\hline SD[ Metric4-Bi/Bi] & 0.0889 & 0.1693 & 0.0443 & 0.0907 & 0.1690 & 0.0480 & 0.0933 & 0.1614 & 0.0328 & 0.0762 \\
\hline SD[Metric4]/SDBi & 0.9297 & 0.8595 & 0.7588 & 0.7418 & 0.8955 & 0.9683 & 0.7269 & 0.8815 & 0.9984 & 0.9280 \\
\hline SD[Metric4-Bi]/SDBi & 0.6382 & 1.2010 & 1.0695 & 1.2695 & 1.3307 & 0.6573 & 1.1059 & 1.2934 & 0.2324 & 0.6018 \\
\hline Mean[Metric5] & 0.0367 & 0.0641 & 0.0168 & 0.0240 & 0.0590 & 0.0207 & 0.0266 & 0.0594 & 0.0351 & 0.0390 \\
\hline SD[Metric5] & 0.0045 & 0.0036 & 0.0005 & 0.0008 & 0.0015 & 0.0015 & 0.0013 & 0.0016 & 0.0049 & 0.0044 \\
\hline Mean[Metric5-Bi] & 0.0008 & 0.0106 & -0.0006 & 0.0001 & 0.0099 & -0.0003 & 0.0005 & 0.0099 & -0.0003 & 0.0006 \\
\hline SD[Metric5-Bi] & 0.0031 & 0.0074 & 0.0008 & 0.0020 & 0.0064 & 0.0010 & 0.0024 & 0.0064 & 0.0011 & 0.0029 \\
\hline Mean[ Metric5-Bi/Bi] & 0.0272 & 0.2178 & -0.0323 & 0.0095 & 0.2204 & -0.0125 & 0.0266 & 0.2182 & -0.0079 & 0.0203 \\
\hline SD[ Metric5-Bi/Bi] & 0.0875 & 0.1630 & 0.0438 & 0.0820 & 0.1534 & 0.0473 & 0.0904 & 0.1514 & 0.0326 & 0.0754 \\
\hline SD[Metric5]/SDBi & 0.8880 & 0.4812 & 0.7154 & 0.4241 & 0.2384 & 0.9467 & 0.5646 & 0.2584 & 0.9932 & 0.8875 \\
\hline SD[Metric5-Bi]/SDBi & 0.6123 & 0.9997 & 1.0437 & 1.0865 & 1.0170 & 0.6443 & 1.0234 & 1.0307 & 0.2310 & 0.5799 \\
\hline Mean[Metric6] & 0.0397 & 0.0708 & 0.0178 & 0.0263 & 0.0638 & 0.0218 & 0.0293 & 0.0649 & 0.0362 & 0.0421 \\
\hline SD[Metric6] & 0.0045 & 0.0037 & 0.0005 & 0.0006 & 0.0013 & 0.0015 & 0.0013 & 0.0016 & 0.0049 & 0.0045 \\
\hline Mean[Metric6-Bi] & 0.0038 & 0.0173 & 0.0004 & 0.0023 & 0.0147 & 0.0008 & 0.0032 & 0.0154 & 0.0008 & 0.0037 \\
\hline SD[Metric6-Bi] & 0.0030 & 0.0074 & 0.0008 & 0.0019 & 0.0065 & 0.0010 & 0.0024 & 0.0064 & 0.0011 & 0.0028 \\
\hline Mean[ Metric6-Bi/Bi] & 0.1133 & 0.3452 & 0.0264 & 0.1037 & 0.3199 & 0.0405 & 0.1317 & 0.3309 & 0.0244 & 0.1026 \\
\hline SD[ Metric6-Bi/Bi] & 0.0927 & 0.1783 & 0.0445 & 0.0852 & 0.1664 & 0.0477 & 0.0975 & 0.1646 & 0.0318 & 0.0780 \\
\hline SD[Metric6]/SDBi & 0.8847 & 0.5020 & 0.6721 & 0.3527 & 0.2081 & 0.9316 & 0.5685 & 0.2513 & 0.9942 & 0.9053 \\
\hline SD[Metric6-Bi]/SDBi & 0.5943 & 0.9926 & 1.0164 & 1.0445 & 1.0224 & 0.6271 & 1.0119 & 1.0309 & 0.2197 & 0.5619 \\
\hline Mean[Metric7] & 0.0453 & 0.0787 & 0.0194 & 0.0286 & 0.0652 & 0.0235 & 0.0322 & 0.0672 & 0.0382 & 0.0464 \\
\hline SD[Metric7] & 0.0054 & 0.0096 & 0.0011 & 0.0029 & 0.0084 & 0.0018 & 0.0033 & 0.0083 & 0.0051 & 0.0056 \\
\hline Mean[Metric7-Bi] & 0.0094 & 0.0252 & 0.0021 & 0.0047 & 0.0161 & 0.0026 & 0.0061 & 0.0177 & 0.0028 & 0.0080 \\
\hline SD[Metric7-Bi] & 0.0040 & 0.0111 & 0.0013 & 0.0034 & 0.0107 & 0.0014 & 0.0037 & 0.0103 & 0.0015 & 0.0041 \\
\hline Mean[ Metric7-Bi/Bi] & 0.2719 & 0.4952 & 0.1211 & 0.2027 & 0.3504 & 0.1244 & 0.2400 & 0.3783 & 0.0822 & 0.2153 \\
\hline SD[ Metric7-Bi/Bi] & 0.1296 & 0.2502 & 0.0748 & 0.1516 & 0.2472 & 0.0685 & 0.1529 & 0.2398 & 0.0447 & 0.1147 \\
\hline SD[Metric7]/SDBi & 1.0552 & 1.2860 & 1.4716 & 1.5567 & 1.3277 & 1.1321 & 1.3880 & 1.3359 & 1.0286 & 1.1352 \\
\hline SD[Metric7-Bi]/SDBi & 0.7828 & 1.4882 & 1.6655 & 1.8702 & 1.6830 & 0.8786 & 1.5918 & 1.6504 & 0.2988 & 0.8170 \\
\hline Mean[Metric8] & 0.0519 & 0.0999 & 0.0221 & 0.0366 & 0.0894 & 0.0261 & 0.0402 & 0.0913 & 0.0409 & 0.0545 \\
\hline SD[Metric8] & 0.0049 & 0.0052 & 0.0005 & 0.0008 & 0.0021 & 0.0015 & 0.0015 & 0.0024 & 0.0050 & 0.0048 \\
\hline Mean[Metric8-Bi] & 0.0160 & 0.0464 & 0.0048 & 0.0126 & 0.0403 & 0.0052 & 0.0141 & 0.0418 & 0.0056 & 0.0160 \\
\hline SD[Metric8-Bi] & 0.0031 & 0.0079 & 0.0008 & 0.0020 & 0.0067 & 0.0010 & 0.0024 & 0.0067 & 0.0011 & 0.0029 \\
\hline Mean[ Metric8-Bi/Bi] & 0.4578 & 0.9001 & 0.2766 & 0.5364 & 0.8504 & 0.2500 & 0.5521 & 0.8719 & 0.1608 & 0.4279 \\
\hline SD[ Metric8-Bi/Bi] & 0.1248 & 0.2519 & 0.0536 & 0.1174 & 0.2344 & 0.0564 & 0.1314 & 0.2326 & 0.0402 & 0.1047 \\
\hline SD[Metric8]/SDBi & 0.9564 & 0.7024 & 0.7008 & 0.4289 & 0.3379 & 0.9560 & 0.6602 & 0.3927 & 1.0030 & 0.9680 \\
\hline SD[Metric8-Bi]/SDBi & 0.6081 & 1.0592 & 1.0270 & 1.0691 & 1.0568 & 0.6330 & 1.0431 & 1.0743 & 0.2218 & 0.5763 \\
\hline Mean[Metric9] & 0.0438 & 0.0742 & 0.0194 & 0.0286 & 0.0628 & 0.0235 & 0.0320 & 0.0648 & 0.0382 & 0.0463 \\
\hline SD[Metric9] & 0.0056 & 0.0104 & 0.0011 & 0.0029 & 0.0096 & 0.0018 & 0.0034 & 0.0096 & 0.0051 & 0.0057 \\
\hline Mean[Metric9-Bi] & 0.0079 & 0.0207 & 0.0021 & 0.0046 & 0.0137 & 0.0026 & 0.0059 & 0.0153 & 0.0028 & 0.0079 \\
\hline SD[Metric9-Bi] & 0.0042 & 0.0120 & 0.0013 & 0.0035 & 0.0116 & 0.0014 & 0.0038 & 0.0113 & 0.0015 & 0.0041 \\
\hline Mean[ Metric9-Bi/Bi] & 0.2282 & 0.4103 & 0.1211 & 0.1992 & 0.2994 & 0.1244 & 0.2352 & 0.3277 & 0.0819 & 0.2129 \\
\hline SD[ Metric9-Bi/Bi] & 0.1321 & 0.2585 & 0.0748 & 0.1540 & 0.2615 & 0.0685 & 0.1569 & 0.2537 & 0.0443 & 0.1157 \\
\hline SD[Metric9]/SDBi & 1.0955 & 1.3945 & 1.4716 & 1.6017 & 1.5098 & 1.1321 & 1.4398 & 1.5414 & 1.0293 & 1.1458 \\
\hline SD[Metric9-Bi]/SDBi & 0.8280 & 1.6187 & 1.6655 & 1.9053 & 1.8328 & 0.8786 & 1.6403 & 1.8094 & 0.2971 & 0.8279 \\
\hline formMean & 0.0111 & 0.0366 & 0.0037 & 0.0110 & 0.0366 & 0.0036 & 0.0110 & 0.0367 & 0.0037 & 0.0110 \\
\hline formStdDev & 0.0021 & 0.0068 & 0.0007 & 0.0020 & 0.0070 & 0.0007 & 0.0020 & 0.0068 & 0.0007 & 0.0021 \\
\hline Points per sphere & 25 & 25 & 25 & 25 & 25 & 25 & 25 & 25 & 25 & 25 \\
\hline Scenario number & 62 & 63 & 64 & 65 & 66 & 67 & 68 & 69 & 70 & 71 \\
\hline
\end{tabular}


18

\begin{tabular}{|c|c|c|c|c|c|c|c|c|c|c|}
\hline Scenario number & 72 & 73 & 74 & 75 & 76 & 77 & 78 & 79 & 80 & 81 \\
\hline IongRangeErrorDist & $\mathrm{N}[0.010,0.010]$ & $\mathrm{N}[0.010,0.001]$ & $\mathrm{N}[0.010,0.001]$ & $\mathrm{N}[0.010,0.001]$ & $\mathrm{N}[0.010,0.003]$ & $\mathrm{N}[0.010,0.003]$ & $\mathrm{N}[0.010,0.003]$ & $\mathrm{N}[0.010,0.010]$ & $\mathrm{N}[0.010,0.010]$ & $\mathrm{N}[0.010,0.010]$ \\
\hline probeRndErrorDist & $\mathrm{N}[0.0015,0.010]$ & $\mathrm{N}[0.005,0.001]$ & $\mathrm{N}[0.005,0.003]$ & $\mathrm{N}[0.005,0.010]$ & $\mathrm{N}[0.005,0.001]$ & $\mathrm{N}[0.005,0.003]$ & $\mathrm{N}[0.005,0.010]$ & $\mathrm{N}[0.005,0.001]$ & $\mathrm{N}[0.005,0.003]$ & $\mathrm{N}[0.005,0.010]$ \\
\hline Mean[Bi] & 0.0568 & 0.0243 & 0.0311 & 0.0560 & 0.0280 & 0.0330 & 0.0564 & 0.0424 & 0.0456 & 0.0636 \\
\hline stdDevBi & 0.0078 & 0.0007 & 0.0019 & 0.0060 & 0.0015 & 0.0022 & 0.0064 & 0.0050 & 0.0050 & 0.0079 \\
\hline Mean[Metric1] & 0.0553 & 0.0232 & 0.0282 & 0.0534 & 0.0273 & 0.0311 & 0.0543 & 0.0417 & 0.0443 & 0.0618 \\
\hline SD[Metric1] & 0.0092 & 0.0009 & 0.0024 & 0.0086 & 0.0015 & 0.0026 & 0.0087 & 0.0050 & 0.0051 & 0.0089 \\
\hline Mean[Metric1-Bi] & -0.0015 & -0.0011 & -0.0029 & -0.0026 & -0.0007 & -0.0019 & -0.0021 & -0.0007 & -0.0013 & -0.0018 \\
\hline SD[Metric1-Bi] & 0.0114 & 0.0011 & 0.0031 & 0.0104 & 0.0011 & 0.0031 & 0.0109 & 0.0012 & 0.0037 & 0.0110 \\
\hline Mean[ Metric1-Bi/Bi] & -0.0118 & -0.0443 & -0.0897 & -0.0357 & -0.0249 & -0.0533 & -0.0255 & -0.0165 & -0.0268 & -0.0158 \\
\hline SD[ Metric1-Bi/Bi] & 0.2026 & 0.0430 & 0.0944 & 0.1823 & 0.0401 & 0.0907 & 0.1909 & 0.0293 & 0.0803 & 0.1719 \\
\hline SD[Metric1]/SDBi & 1.1787 & 1.1663 & 1.2748 & 1.4351 & 0.9699 & 1.1870 & 1.3516 & 0.9913 & 1.0208 & 1.1323 \\
\hline SD[Metric1-Bi]/SDBi & 1.4661 & 1.4556 & 1.6339 & 1.7414 & 0.7387 & 1.4219 & 1.7080 & 0.2492 & 0.7366 & 1.4048 \\
\hline Mean[Metric2] & 0.0787 & 0.0250 & 0.0347 & 0.0778 & 0.0288 & 0.0372 & 0.0789 & 0.0432 & 0.0497 & 0.0847 \\
\hline SD[Metric2] & 0.0032 & 0.0005 & 0.0005 & 0.0009 & 0.0014 & 0.0012 & 0.0012 & 0.0049 & 0.0045 & 0.0032 \\
\hline Mean[Metric2-Bi] & 0.0218 & 0.0007 & 0.0036 & 0.0218 & 0.0009 & 0.0042 & 0.0225 & 0.0008 & 0.0041 & 0.0211 \\
\hline SD[Metric2-Bi] & 0.0075 & 0.0008 & 0.0019 & 0.0060 & 0.0010 & 0.0022 & 0.0065 & 0.0011 & 0.0030 & 0.0076 \\
\hline Mean[ Metric2-Bi/Bi] & 0.4071 & 0.0290 & 0.1197 & 0.4056 & 0.0323 & 0.1312 & 0.4160 & 0.0190 & 0.0939 & 0.3493 \\
\hline SD[ Metric2-Bi/Bi] & 0.1768 & 0.0324 & 0.0676 & 0.1468 & 0.0351 & 0.0721 & 0.1554 & 0.0256 & 0.0699 & 0.1536 \\
\hline SD[Metric2]/SDBi & 0.4096 & 0.6598 & 0.2896 & 0.1496 & 0.8796 & 0.5712 & 0.1825 & 0.9864 & 0.8956 & 0.4123 \\
\hline SD[Metric2-Bi]/SDBi & 0.9577 & 1.0598 & 1.0369 & 1.0071 & 0.6261 & 1.0075 & 1.0075 & 0.2119 & 0.5932 & 0.9664 \\
\hline Mean[Metric3] & 0.0548 & 0.0230 & 0.0269 & 0.0497 & 0.0271 & 0.0302 & 0.0509 & 0.0417 & 0.0438 & 0.0595 \\
\hline SD[Metric3] & 0.0078 & 0.0008 & 0.0021 & 0.0067 & 0.0015 & 0.0024 & 0.0068 & 0.0050 & 0.0052 & 0.0078 \\
\hline Mean[Metric3-Bi] & -0.0020 & -0.0013 & -0.0042 & -0.0063 & -0.0009 & -0.0028 & -0.0055 & -0.0008 & -0.0018 & -0.0040 \\
\hline SD[Metric3-Bi] & 0.0103 & 0.0011 & 0.0028 & 0.0090 & 0.0011 & 0.0029 & 0.0095 & 0.0012 & 0.0036 & 0.0101 \\
\hline Mean[ Metric3-Bi/Bi] & -0.0206 & -0.0539 & -0.1324 & -0.1024 & -0.0304 & -0.0833 & -0.0868 & -0.0180 & -0.0368 & -0.0523 \\
\hline SD[ Metric3-Bi/Bi] & 0.1805 & 0.0421 & 0.0853 & 0.1507 & 0.0398 & 0.0853 & 0.1593 & 0.0293 & 0.0790 & 0.1536 \\
\hline SD[Metric3]/SDBi & 1.0024 & 1.1460 & 1.1244 & 1.1213 & 0.9783 & 1.1220 & 1.0622 & 0.9961 & 1.0310 & 0.9910 \\
\hline SD[Metric3-Bi]/SDBi & 1.3179 & 1.4321 & 1.5136 & 1.4962 & 0.7356 & 1.3575 & 1.4765 & 0.2490 & 0.7284 & 1.2843 \\
\hline Mean[Metric4] & 0.0566 & 0.0232 & 0.0280 & 0.0527 & 0.0273 & 0.0310 & 0.0536 & 0.0419 & 0.0445 & 0.0620 \\
\hline SD[Metric4] & 0.0064 & 0.0005 & 0.0012 & 0.0052 & 0.0014 & 0.0018 & 0.0052 & 0.0050 & 0.0049 & 0.0061 \\
\hline Mean[Metric4-Bi] & -0.0002 & -0.0011 & -0.0030 & -0.0032 & -0.0007 & -0.0020 & -0.0028 & -0.0006 & -0.0012 & -0.0016 \\
\hline SD[Metric4-Bi] & 0.0092 & 0.0008 & 0.0022 & 0.0079 & 0.0010 & 0.0025 & 0.0081 & 0.0011 & 0.0032 & 0.0090 \\
\hline Mean[ Metric4-Bi/Bi] & 0.0119 & -0.0439 & -0.0949 & -0.0471 & -0.0246 & -0.0584 & -0.0386 & -0.0132 & -0.0232 & -0.0125 \\
\hline SD[ Metric4-Bi/Bi] & 0.1619 & 0.0321 & 0.0661 & 0.1343 & 0.0349 & 0.0716 & 0.1388 & 0.0260 & 0.0682 & 0.1381 \\
\hline SD[Metric4]/SDBi & 0.8171 & 0.7462 & 0.6323 & 0.8676 & 0.9185 & 0.8339 & 0.8163 & 0.9955 & 0.9704 & 0.7766 \\
\hline SD[Metric4-Bi]/SDBi & 1.1768 & 1.1064 & 1.1937 & 1.3121 & 0.6505 & 1.1493 & 1.2697 & 0.2213 & 0.6329 & 1.1443 \\
\hline Mean[Metric5] & 0.0663 & 0.0235 & 0.0297 & 0.0627 & 0.0275 & 0.0326 & 0.0635 & 0.0420 & 0.0456 & 0.0707 \\
\hline SD[Metric5] & 0.0037 & 0.0005 & 0.0009 & 0.0017 & 0.0014 & 0.0015 & 0.0020 & 0.0050 & 0.0047 & 0.0040 \\
\hline Mean[Metric5-Bi] & 0.0095 & -0.0008 & -0.0013 & 0.0068 & -0.0005 & -0.0004 & 0.0070 & -0.0004 & 0.0000 & 0.0072 \\
\hline SD[Metric5-Bi] & 0.0077 & 0.0008 & 0.0020 & 0.0062 & 0.0010 & 0.0023 & 0.0066 & 0.0011 & 0.0031 & 0.0079 \\
\hline Mean[ Metric5-Bi/Bi] & 0.1866 & -0.0335 & -0.0399 & 0.1338 & -0.0173 & -0.0096 & 0.1383 & -0.0099 & 0.0029 & 0.1268 \\
\hline SD[ Metric5-Bi/Bi] & 0.1537 & 0.0318 & 0.0625 & 0.1212 & 0.0349 & 0.0689 & 0.1279 & 0.0260 & 0.0682 & 0.1327 \\
\hline SD[Metric5]/SDBi & 0.4751 & 0.7272 & 0.4572 & 0.2813 & 0.9116 & 0.7137 & 0.3195 & 0.9931 & 0.9435 & 0.5117 \\
\hline SD[Metric5-Bi]/SDBi & 0.9933 & 1.0888 & 1.0956 & 1.0321 & 0.6469 & 1.0731 & 1.0328 & 0.2210 & 0.6249 & 1.0006 \\
\hline Mean[Metric6] & 0.0732 & 0.0246 & 0.0320 & 0.0677 & 0.0286 & 0.0352 & 0.0693 & 0.0431 & 0.0487 & 0.0776 \\
\hline SD[Metric6] & 0.0039 & 0.0005 & 0.0007 & 0.0014 & 0.0014 & 0.0014 & 0.0018 & 0.0050 & 0.0047 & 0.0041 \\
\hline Mean[Metric6-Bi] & 0.0164 & 0.0002 & 0.0010 & 0.0118 & 0.0006 & 0.0022 & 0.0128 & 0.0007 & 0.0031 & 0.0140 \\
\hline SD[Metric6-Bi] & 0.0076 & 0.0008 & 0.0020 & 0.0061 & 0.0010 & 0.0023 & 0.0066 & 0.0011 & 0.0030 & 0.0078 \\
\hline Mean[ Metric6-Bi/Bi] & 0.3096 & 0.0105 & 0.0353 & 0.2241 & 0.0219 & 0.0690 & 0.2427 & 0.0161 & 0.0716 & 0.2356 \\
\hline SD[ Metric6-Bi/Bi] & 0.1655 & 0.0324 & 0.0642 & 0.1292 & 0.0350 & 0.0706 & 0.1382 & 0.0254 & 0.0689 & 0.1429 \\
\hline SD[Metric6]/SDBi & 0.4958 & 0.6949 & 0.3647 & 0.2381 & 0.9027 & 0.6664 & 0.2785 & 0.9937 & 0.9382 & 0.5269 \\
\hline SD[Metric6-Bi]/SDBi & 0.9711 & 1.0742 & 1.0592 & 1.0211 & 0.6302 & 1.0394 & 1.0248 & 0.2117 & 0.6012 & 0.9888 \\
\hline Mean[Metric7] & 0.0793 & 0.0264 & 0.0354 & 0.0699 & 0.0305 & 0.0389 & 0.0717 & 0.0451 & 0.0534 & 0.0833 \\
\hline SD[Metric7] & 0.0097 & 0.0011 & 0.0029 & 0.0091 & 0.0016 & 0.0032 & 0.0092 & 0.0050 & 0.0057 & 0.0103 \\
\hline Mean[Metric7-Bi] & 0.0225 & 0.0020 & 0.0043 & 0.0139 & 0.0025 & 0.0059 & 0.0152 & 0.0027 & 0.0078 & 0.0198 \\
\hline SD[Metric7-Bi] & 0.0115 & 0.0013 & 0.0035 & 0.0109 & 0.0013 & 0.0036 & 0.0113 & 0.0014 & 0.0041 & 0.0120 \\
\hline Mean[ Metric7-Bi/Bi] & 0.4183 & 0.0844 & 0.1425 & 0.2622 & 0.0919 & 0.1818 & 0.2861 & 0.0647 & 0.1748 & 0.3267 \\
\hline SD[ Metric7-Bi/Bi] & 0.2390 & 0.0538 & 0.1154 & 0.2071 & 0.0484 & 0.1141 & 0.2186 & 0.0355 & 0.0983 & 0.2108 \\
\hline SD[Metric7]/SDBi & 1.2514 & 1.4923 & 1.5309 & 1.5230 & 1.0620 & 1.4800 & 1.4422 & 1.0002 & 1.1315 & 1.3174 \\
\hline SD[Metric7-Bi]/SDBi & 1.4757 & 1.7438 & 1.8490 & 1.8113 & 0.8435 & 1.6617 & 1.7645 & 0.2824 & 0.8264 & 1.5252 \\
\hline Mean[Metric8] & 0.1034 & 0.0291 & 0.0432 & 0.0956 & 0.0331 & 0.0467 & 0.0979 & 0.0478 & 0.0615 & 0.1093 \\
\hline SD[Metric8] & 0.0053 & 0.0005 & 0.0008 & 0.0021 & 0.0014 & 0.0016 & 0.0026 & 0.0050 & 0.0049 & 0.0054 \\
\hline Mean[Metric8-Bi] & 0.0466 & 0.0048 & 0.0122 & 0.0397 & 0.0051 & 0.0137 & 0.0415 & 0.0054 & 0.0158 & 0.0457 \\
\hline SD[Metric8-Bi] & 0.0080 & 0.0008 & 0.0020 & 0.0063 & 0.0010 & 0.0023 & 0.0068 & 0.0011 & 0.0031 & 0.0082 \\
\hline Mean[ Metric8-Bi/Bi] & 0.8496 & 0.1977 & 0.3971 & 0.7283 & 0.1851 & 0.4209 & 0.7563 & 0.1284 & 0.3532 & 0.7406 \\
\hline SD[ Metric8-Bi/Bi] & 0.2336 & 0.0370 & 0.0851 & 0.1828 & 0.0400 & 0.0909 & 0.1956 & 0.0308 & 0.0873 & 0.2000 \\
\hline SD[Metric8]/SDBi & 0.6780 & 0.7101 & 0.4108 & 0.3529 & 0.9165 & 0.7341 & 0.4002 & 0.9985 & 0.9851 & 0.6851 \\
\hline SD[Metric8-Bi]/SDBi & 1.0232 & 1.0808 & 1.0753 & 1.0502 & 0.6330 & 1.0668 & 1.0589 & 0.2127 & 0.6125 & 1.0381 \\
\hline Mean[Metric9] & 0.0767 & 0.0264 & 0.0354 & 0.0695 & 0.0305 & 0.0389 & 0.0713 & 0.0451 & 0.0534 & 0.0830 \\
\hline SD[Metric9] & 0.0108 & 0.0011 & 0.0029 & 0.0095 & 0.0016 & 0.0032 & 0.0096 & 0.0050 & 0.0057 & 0.0107 \\
\hline Mean[Metric9-Bi] & 0.0199 & 0.0020 & 0.0043 & 0.0136 & 0.0025 & 0.0059 & 0.0148 & 0.0027 & 0.0078 & 0.0194 \\
\hline SD[Metric9-Bi] & 0.0125 & 0.0013 & 0.0035 & 0.0111 & 0.0013 & 0.0036 & 0.0117 & 0.0014 & 0.0041 & 0.0122 \\
\hline Mean[ Metric9-Bi/Bi] & 0.3709 & 0.0844 & 0.1425 & 0.2562 & 0.0919 & 0.1818 & 0.2790 & 0.0645 & 0.1748 & 0.3214 \\
\hline SD[ Metric9-Bi/Bi] & 0.2525 & 0.0538 & 0.1154 & 0.2115 & 0.0484 & 0.1141 & 0.2244 & 0.0355 & 0.0983 & 0.2144 \\
\hline SD[Metric9]/SDBi & 1.3943 & 1.4923 & 1.5309 & 1.5769 & 1.0620 & 1.4800 & 1.5044 & 1.0022 & 1.1320 & 1.3581 \\
\hline SD[Metric9-Bi]/SDBi & 1.6082 & 1.7438 & 1.8490 & 1.8580 & 0.8435 & 1.6617 & 1.8249 & 0.2831 & 0.8270 & 1.5594 \\
\hline formMean & 0.0366 & 0.0037 & 0.0109 & 0.0368 & 0.0037 & 0.0108 & 0.0366 & 0.0036 & 0.0110 & 0.0367 \\
\hline formStdDev & 0.0070 & 0.0007 & 0.0020 & 0.0068 & 0.0007 & 0.0020 & 0.0068 & 0.0007 & 0.0021 & 0.0068 \\
\hline Points per sphere & 25 & 25 & 25 & 25 & 25 & 25 & 25 & 25 & 25 & 25 \\
\hline Scenario number & 72 & 73 & 74 & 75 & 76 & 77 & 78 & 79 & 80 & 81 \\
\hline
\end{tabular}

